\title{
Dysfunction of parvalbumin neurons in the cerebellar nuclei produces an action tremor
}

\author{
Mu Zhou, ${ }^{1}$ Maxwell D. Melin, ${ }^{1}$ Wei Xu, ${ }^{1}$ and Thomas C. Südhof ${ }^{1,2}$ \\ 'Department of Molecular and Cellular Physiology and ${ }^{2}$ Howard Hughes Medical Institute, Stanford University School of Medicine, Stanford, California, USA
}

\begin{abstract}
Essential tremor is a common brain disorder affecting millions of people, yet the neuronal mechanisms underlying this prevalent disease remain elusive. Here, we showed that conditional deletion of synaptotagmin-2, the fastest $\mathrm{Ca}^{2+}$ sensor for synaptic neurotransmitter release, from parvalbumin neurons in mice caused an action tremor syndrome resembling the core symptom of essential tremor patients. Combining brain region-specific and cell type-specific genetic manipulation methods, we found that deletion of synaptotagmin-2 from excitatory parvalbumin-positive neurons in cerebellar nuclei was sufficient to generate an action tremor. The synaptotagmin-2 deletion converted synchronous into asynchronous neurotransmitter release in projections from cerebellar nuclei neurons onto gigantocellular reticular nucleus neurons, which might produce an action tremor by causing signal oscillations during movement. The tremor was rescued by completely blocking synaptic transmission with tetanus toxin in cerebellar nuclei, which also reversed the tremor phenotype in the traditional harmalineinduced essential tremor model. Using a promising animal model for action tremor, our results thus characterized a synaptic circuit mechanism that may underlie the prevalent essential tremor disorder.
\end{abstract}

\section{Introduction}

Essential tremor is the most common movement disorder of human patients (1). Clinically, essential tremor is characterized by a $4-\mathrm{Hz}$ to $12-\mathrm{Hz}$ action tremor (2) that affects different body parts, making basic daily activities difficult if not impossible in severe cases. Although no consensus has been reached on its exact prevalence, surveys show that the disease is present in $4 \%$ of individuals aged 40 and up (3). Essential tremor prevalence increases with age, such that up to $14 \%$ of people aged 65 and above exhibit essential tremor (4). Despite its widespread presence, our understanding of essential tremor's biological mechanisms remains very limited (5), and few therapeutic options are available.

Studies of the etiology of essential tremor traditionally focused on the inferior olive (6-8). In an animal model of essential tremor induced by i.p. injections of harmaline, rhythmic burst-firing was detected in inferior olive neurons, and an action tremor was induced that exhibited the same frequency as the rhythmic firing in inferior olive neurons (6). Although these findings were promising, decades of clinical research found little evidence for a dysfunction or for pathological changes in the inferior olive in essential tremor patients (5). More recently, postmortem analyses and advanced neuroimaging techniques have identified pathological changes in the cerebellum, including Purkinje cells, basket cells, and the cerebellar nuclei $(\mathrm{CBN})$ in essential tremor patients (9-13). In particular, degeneration of Purkinje cells has become a widely believed hypothesis to account for essential tremor (5). Yet, the data so far are correlative. There is no evidence to show the causality between

Conflict of interest: The authors have declared that no conflict of interest exists. Copyright: () 2020, American Society for Clinical Investigation.

Submitted: December 17, 2019; Accepted: June 24, 2020; Published: August 24, 2020.

Reference information: J Clin Invest. 2020;130(10):5142-5156.

https://doi.org/10.1172/JCl135802. cerebellar pathologies and essential tremor symptoms. More importantly, the physiological basis by which cerebellar dysfunction might lead to essential tremor remains unknown.

A major obstacle to a better understanding of the etiology of essential tremor is the lack of a reliable genetic animal model (14-16). The traditional harmaline-induced essential tremor model is useful for the preclinical testing of antitremor drugs. However, this model has limited value for translational research since its tremor symptoms spontaneously resolve in a few hours $(6,8)$. Recently, several genetic tremor animal models were described (15-18) that provided critical insights into the neural mechanisms of essential tremor. Nevertheless, these genetic models have limitations because they either exhibit nonspecific action tremor or produce a much higher tremor frequency than observed in essential tremor patients.

Synaptotagmin-2 (Syt2) is an ultrafast $\mathrm{Ca}^{2+}$ sensor for fast neurotransmitter release (19-21). We initially crossed Syt 2 conditional knockout $\left(S y t 2^{\mathrm{fl} / \mathrm{fl}}\right)$ mice with parvalbumin-Cre (PV $\left.{ }^{\text {cre }}\right)$ driver mice in order to study the functional roles of Syt2 at the calyx of Held synapse (21) and at cortical inhibitory synapses. Unexpectedly, we found that $\mathrm{PV}^{\text {cre }} \mathrm{Syt}^{\mathrm{ft} / \mathrm{fl}}$ mice exhibited a robust action tremor phenotype without other obvious behavioral abnormalities. This surprising discovery provided us a unique opportunity to examine the circuit components and pathological synaptic release properties that can underlie action tremor, the core symptom of the prevalent essential tremor disorder in human patients.

Combining region-, cell type-, and projection-specific deletions of Syt2, we identified the dysfunction of excitatory $\mathrm{PV}^{+}$neurons in the $\mathrm{CBN}$ that project to neurons in the brainstem as the cause of action tremor. We determined that in $\mathrm{PV}^{\text {cre }}$ Syt $2^{\mathrm{f} / / \mathrm{fl}}$ mice, fast synchronous synaptic neurotransmitter release in this cerebellum $\rightarrow$ brainstem circuit was converted into asynchronous neurotransmitter release, which then led to action tremor. We also found that blocking synaptic transmission in the $\mathrm{CBN}$ reversed the action trem- 
Tremor quantification
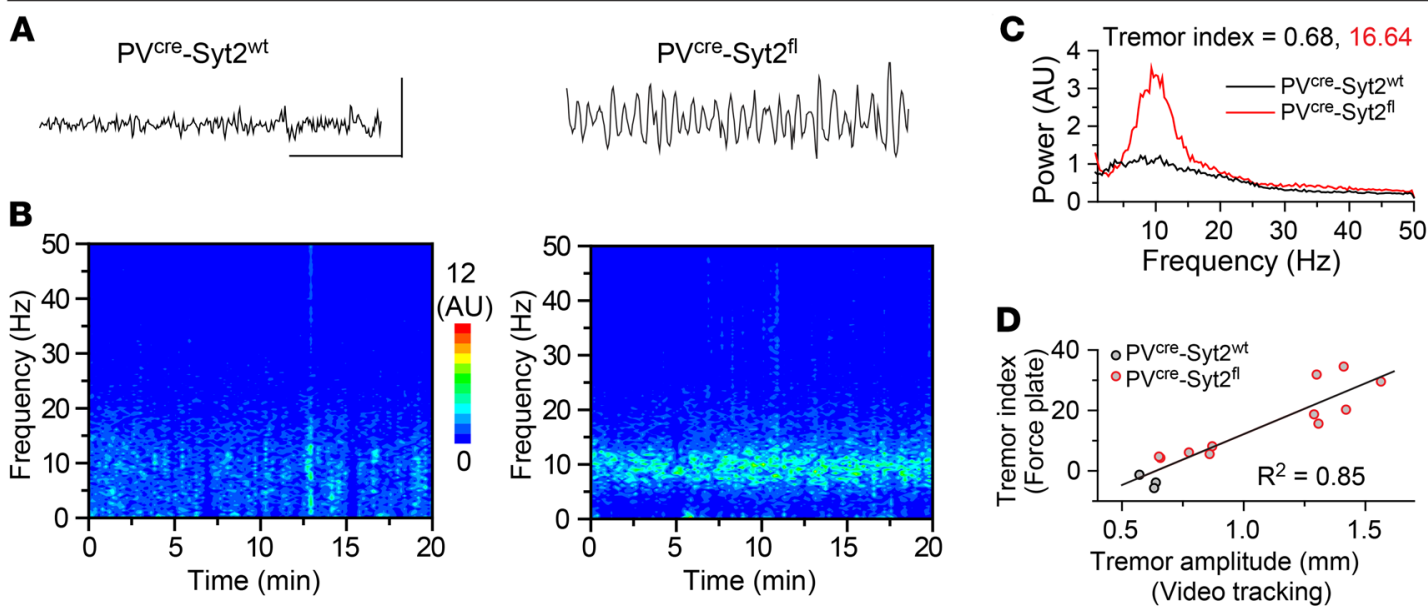

Characterization of PVcre-Syt2 ${ }^{\mathrm{fl}}$ mice
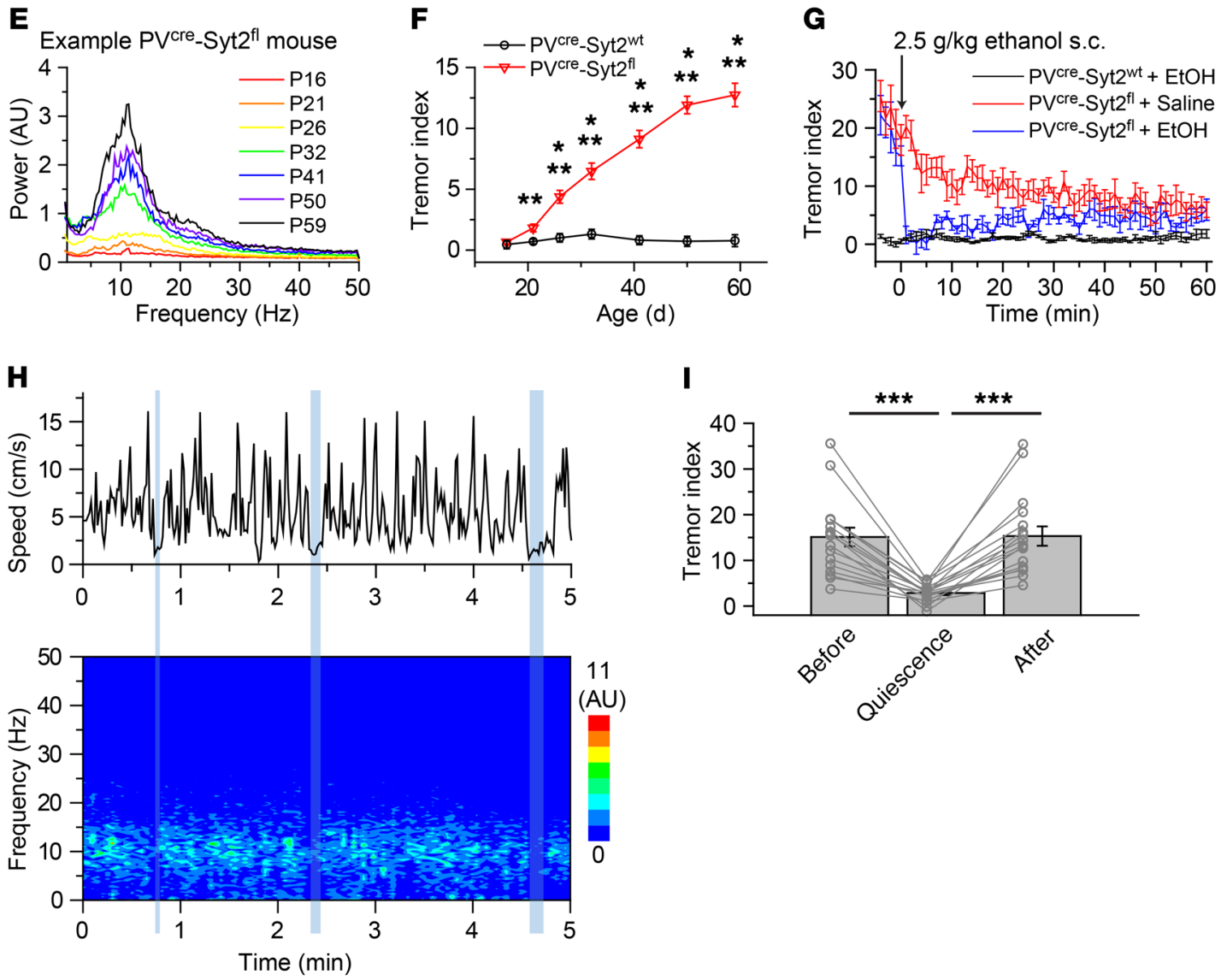

Figure 1. PV're Syt2 ${ }^{\text {fil }}$ mice as an animal model for action tremor. (A-C) Exemplary tremor recordings on a force plate to illustrate the tremor observed in PV cre Syt2 ${ }^{f f}$ mice compared with PV cre Syt2 ${ }^{\text {wt }}$ mice. (A) Raw data of 3-second weight measurement using the force-plate actometer. (B) Spectrograph calculated from 20-minute force-plate measurements. (C) Averaged power spectrum from data in B. The tremor index was calculated by integrating the power in the $9-\mathrm{Hz}$ to $12-\mathrm{Hz}$ range and using the averaged power in the $3-\mathrm{Hz}$ to $6-\mathrm{Hz}$ range as baseline. (D) Correlation of the tremor index measured on a force plate and the tremor amplitude monitored simultaneously by video tracking in PVcre Syt2 ${ }^{\mathrm{fl}}$ and control mice at different ages. (E) Power spectra of forceplate measurements from a representative PVcre Syt ${ }^{f 1}$ mouse at different ages. (F) Summary plot of the tremor index of PV cre Syt2 ${ }^{\text {wt }}$ and PVcre Syt ${ }^{f 1}$ mice as a function of age $\left(n=19 \mathrm{PV}^{\text {cre }}\right.$ Syt2 $2^{\mathrm{wt}}, n=18 \mathrm{PV}^{\mathrm{cre}}$ Syt2 $\left.2^{\mathrm{f}}\right)$. (C) Summary plot of the tremor index of PV cre Syt ${ }^{\mathrm{ft}}$ and control mice as a function of time after an s.c. injection of ethanol (EtOH); PV ${ }^{\text {cre }}$ Syt2 $2^{\mathrm{fl}}$ mice injected with saline used as a further control $\left(n=5\right.$ control + EtOH, $n=7 \mathrm{PV}^{\text {cre }}$ Syt2 $2^{\mathrm{fl}}+$ saline, $n=6 \mathrm{PV}^{\mathrm{cre}}$ Syt2 $\left.{ }^{f l}+\mathrm{EtOH}\right)$. (H) Exemplary simultaneous measurements of the movements and tremor in a PVcre Syt ${ }^{f 1}$ mouse. Episodes longer than 3 seconds with speed less than $2.5 \mathrm{~cm} / \mathrm{s}$ are indicated by vertical blue shaded bars. (I) Summary graph of the tremor index of PV ${ }^{\text {cre }}$ Syt2 $2^{\mathrm{fl}}$ mice before, during, and after periods of quiescence lasting longer than 3 seconds $(n=17)$. For $\mathbf{F}, \mathbf{G}$, and $\mathbf{I}$, data are shown as means \pm SEM from at least 3 independent litters. ${ }^{*} P<0.05$ ${ }^{* *} P<0.01 ;{ }^{* *} P<0.001$ by 2 -sided, unpaired $t$ test $(\mathbf{F})$ or 1-way ANOVA (I). Scale bars: $20 \mathrm{~g}$ (A, vertical); $1 \mathrm{~s}$ (A, horizontal). 
or phenotype in $\mathrm{PV}^{\text {cre }}$ Syt $2^{\mathrm{fl} / \mathrm{fl}}$ mice, and that this rescue approach was also effective for harmaline-induced action tremor, the traditional animal model of essential tremor. Based on these data, we propose a conceptual framework to explain how temporally delayed synaptic transmission in the cerebellum $\rightarrow$ brainstem pathway could generate an action tremor. In summary, our study validated a promising genetic mouse model for action tremor and defined critical circuit components that generate such a tremor. The circuit components and synaptic defects we identified may provide significant insights into the etiology and therapeutic intervention of the widespread essential tremor brain disorder.

\section{Results}

$P V^{\text {cre }} S y t 2^{f l}$ mice are a promising animal model for action tremor. By crossing $\mathrm{PV}^{\text {cre/cre }}$ Syt $2^{\mathrm{fl} /+}$ mice with Syt $2^{\mathrm{fl} / \mathrm{fl}}$ mice, we generated mice with a deletion of Syt2 in PV neurons (PV ${ }^{\text {cre/+ }}$ Syt $2^{\mathrm{fl} / \mathrm{fl}}$, referred to as $\mathrm{PV}^{\text {cre }}$ Syt $2^{\mathrm{fl}}$ ) that exhibited a robust action tremor phenotype, while their heterozygous littermates ( $\mathrm{PV}^{\mathrm{cre} /+} \mathrm{Syt} 2^{\mathrm{fl} /+}$, referred to as $\mathrm{PV}^{\text {cre }}$ Syt $2^{\mathrm{wt}}$ or control) were normal (Supplemental Videos 1 and 2; supplemental material available online with this article; https:/doi.org/10.1172/JCI135802DS1). Immunostaining confirmed the deletion of Syt 2 from $\mathrm{PV}^{+}$synapses (Supplemental Figure 1). To quantify the action tremor, we used a $28 \times 28 \mathrm{~cm}$ force-plate actometer that was designed to measure the whole body weight at $100 \mathrm{~Hz}$ (22). PV ${ }^{\text {cre }}$ Syt $2^{\mathrm{fl}}$ mice exhibited an obvious tremor with an approximately $10-\mathrm{Hz}$ rhythm, whereas their littermate $\mathrm{PV}^{\text {cre }}$ Syt $2^{\text {wt }}$ controls did not (Figure $1, \mathrm{~A}$ and B). For each mouse, we plotted the averaged power spectrum and defined a "tremor index" as the summation of power in the $9-\mathrm{Hz}$ to $12-\mathrm{Hz}$ range, using the power in the $3-\mathrm{Hz}$ to $6-\mathrm{Hz}$ range as the baseline (Figure 1C). To cross-validate this tremor quantification method, we also directly measured the tremor amplitude of $\mathrm{PV}^{\mathrm{cre}} \mathrm{Syt}^{\mathrm{fl}}$ and control mice using video tracking methods, while at the same time measuring their behavior on the force plate. Using a high-speed camera and video tracking software, the detailed movements of mice were recorded and band-pass filtered $(9-12 \mathrm{~Hz})$ to calculate the tremor amplitude (Supplemental Figure 2). This approach confirmed the tremor phenotype in $\mathrm{PV}^{\mathrm{cre}}$ Syt ${ }^{\mathrm{fl}}$ mice and validated the usage of the force-plate actometer to quantify the tremor phenotype (Figure 1D).

The tremor of $\mathrm{PV}^{\text {cre }}$ Syt $2^{\mathrm{fl}}$ mice manifested at the time of weaning and became increasingly stronger as the mice grew older, as documented by force-plate quantifications as a function of age (Figure 1, E and F). Importantly, the observed age-dependent

\section{Table 1. Characteristics of PVcre Syt2 ${ }^{\mathrm{fl}}$ mouse model}

\begin{tabular}{lccc} 
Characteristic & ET patients & Harmaline mouse model & PV $^{\text {cre }}$ Syt2 ${ }^{\mathrm{fl}}$ mouse model \\
\hline Frequency $(\mathrm{Hz})$ & $4-12$ & $11-14^{A}$ & $9-12$ \\
\hline Acute/Chronic & Chronic & Acute & Chronic \\
Progressive & Yes & No $^{A}$ & Yes \\
Alcohol & Effective & Effective & Effective \\
Tremor type & Action & Action & Action \\
Brain region & Cerebellum & Inferior olive ${ }^{A}$ & Cerebellum
\end{tabular}

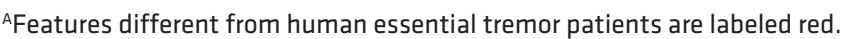

increase of tremor strength was not due to the increase in body size, as shown by plotting the tremor index normalized to the body weight (Supplemental Figure 3A). This age-dependent pattern of increasing tremor is consistent with clinical observations (2). Besides the tremor phenotype, $\mathrm{PV}^{\text {cre }}$ Syt $2^{\mathrm{fl}}$ mice were grossly normal. Their body weight was similar to that of littermate controls (Supplemental Figure 3B). They were fertile and exhibited a normal lifespan, as followed up to 1.3 years, the oldest age we monitored. $\mathrm{PV}^{\text {cre }}$ Syt $2^{\mathrm{fl}}$ mice likely had normal cognitive abilities as suggested by results from spontaneous alternating Y maze (Supplemental Figure 3C), novel object recognition (Supplemental Figure 3D), and fear conditioning assays (Supplemental Figure $3 \mathrm{E}) . \mathrm{PV}^{\text {cre }}$ Syt $2^{\mathrm{fl}}$ mice did, however, show a significant deficit in the rotarod test (Supplemental Figure 3F), which was not surprising given their striking action tremor.

We next tested whether ethanol, which has been shown to temporarily alleviate essential tremor symptoms in human patients (2, 23), had a similar effect in $\mathrm{PV}^{\mathrm{cre}} \mathrm{Syt} 2^{\mathrm{fl}}$ mice. A single s.c. injection of $2.5 \mathrm{~g} / \mathrm{kg}$ ethanol dramatically but temporarily suppressed the tremor of $\mathrm{PV}^{\text {cre }}$ Syt $2^{\mathrm{fl}}$ mice (Figure $1 \mathrm{G}$ and Supplemental Figure $3 G$ ). Importantly, the suppression of the tremor by ethanol could not be explained by the reduction in locomotion (Supplemental Figure 3, $\mathrm{H}$ and $\mathrm{I}$ ). Moreover, we noticed that the $\mathrm{PV}^{\text {cre }}$ Syt $2^{\mathrm{fl}}$ mice had little or no tremor when they were at rest and not moving (Supplemental Videos 2 and 3), suggesting that they had an action tremor phenotype. We quantified this aspect by measuring the moving speed and the instantaneous tremor index of $\mathrm{PV}^{\text {cre }}$ Syt $2^{\mathrm{fl}}$ mice at the same time (Figure $1 \mathrm{H}$ ). Although the mice usually continuously explored the novel environment and had few quiescent episodes, their action tremor phenotype became obvious when we selected all quiescent epochs of longer than 3 seconds and examined the tremor index before, during, and after these quiescent episodes (Figure 1I). Clearly, the tremor decreased during a mouse's quiescent period. The action tremor phenotype of $\mathrm{PV}^{\text {cre }}$ Syt $2^{\mathrm{fl}}$ mice was further illustrated in fear conditioning assays, during which PV ${ }^{\text {cre }}$ Syt $2^{\text {fl }}$ mice substantially froze to contextual cues (Supplemental Video 4), strongly suggesting lack of a resting tremor. Together, these results indicate that $\mathrm{PV}^{\mathrm{cre}}$ Syt $2^{\mathrm{fl}}$ mice are a reliable genetic animal model for action tremor and a promising candidate to model human essential tremor disorder (Table 1).

Syt 2 deletion from the cerebellum is sufficient to generate an action tremor. To identify the brain region that is responsible for generating the action tremor in $\mathrm{PV}^{\text {cre }}$ Syt $2^{\mathrm{fl}}$ mice, we first compared the Syt2 expression levels in $\mathrm{PV}^{\mathrm{cre}}$ and $\mathrm{PV}^{\mathrm{cre}}$ Syt $2^{\mathrm{fl}}$ mice across the entire brain (Figure 2, A and B, and Supplemental Figure

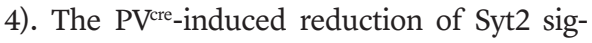
nals was obvious in the cortex, hippocampus, and cerebellum (Figure 2, A and B). Since the reduction of Syt 2 signals was most dramatic in cortical areas, likely due to the colocalization of Syt2 and PV expression (24), we analyzed the effect of the Syt2 deletion on inhibitory synaptic responses in the medial prefrontal cortex (mPFC) using whole-cell patch-clamp recordings in acute slices. We tested the change of spontaneous inhibitory postsynaptic currents (sIPSCs) by injecting adeno-associated viruses 

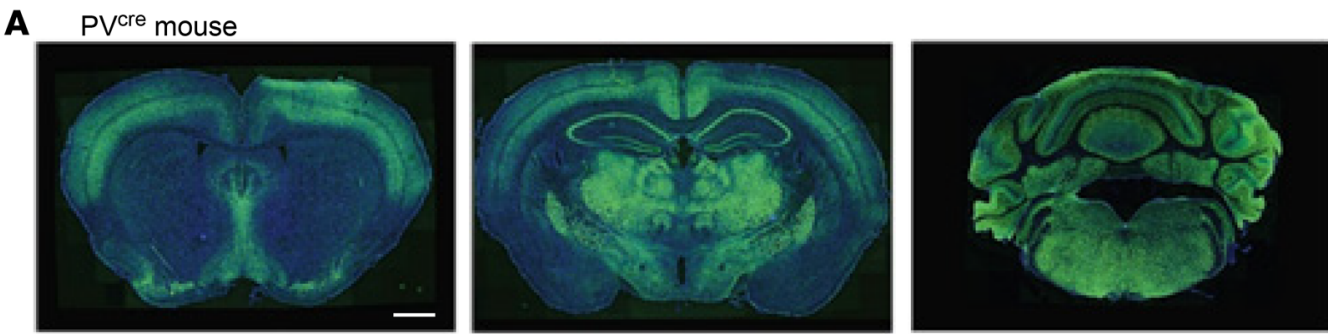

B PVcre_Syt2 $2^{f l}$ mouse
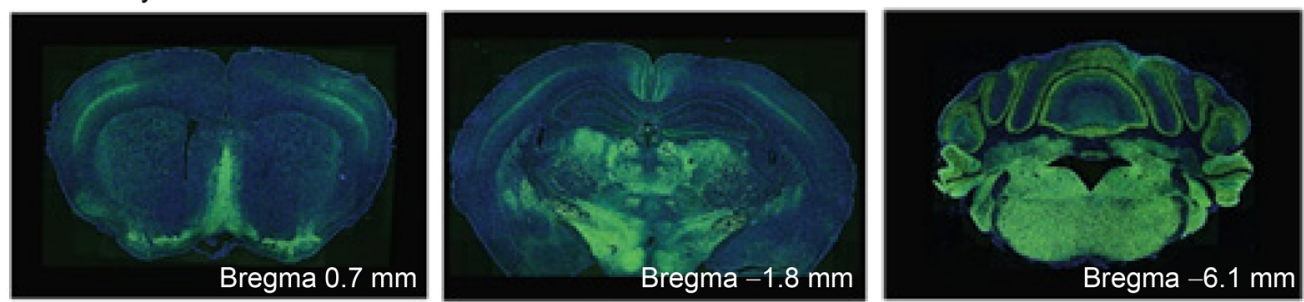

Slice physiology in MPFC

C

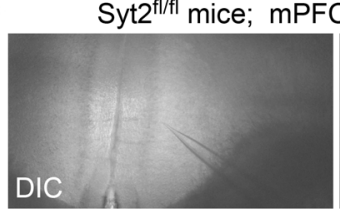

Non-GFP side

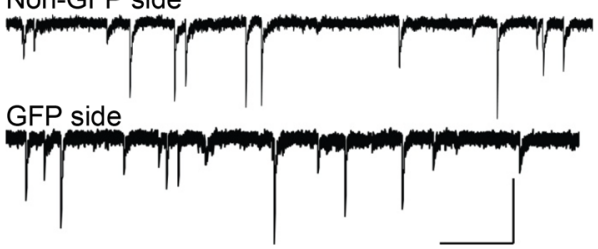

E

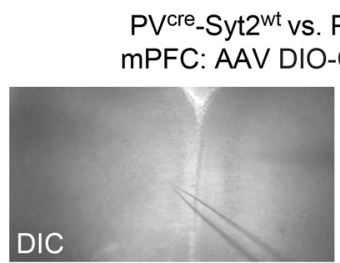

Lase

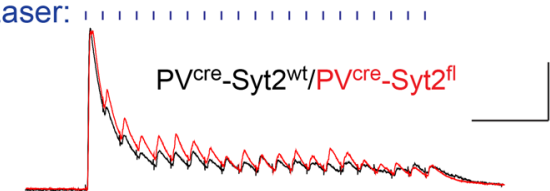

sIPSC frequency
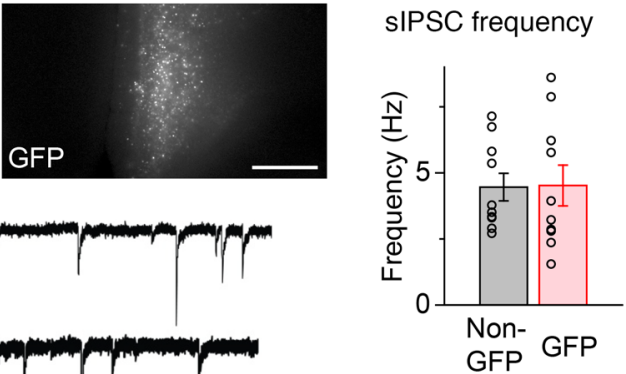

GFP

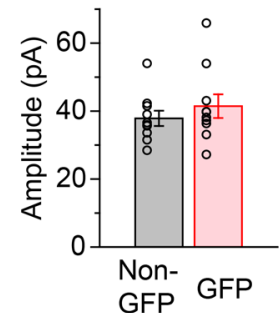

F Evoked IPSC amplitude

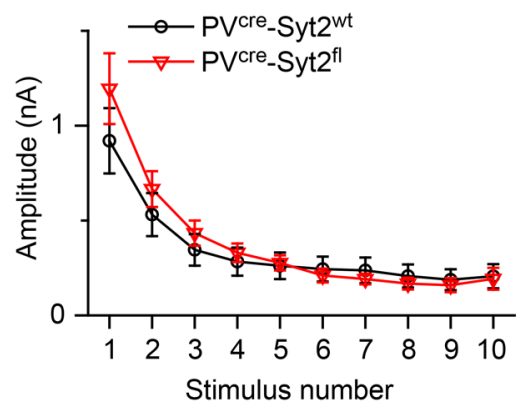

Figure 2. Syt2 is prominently expressed in cortical PV+ neurons, but deletion of Syt2 from mPFC PV+ neurons does not impair their synaptic releases. (A and B) Representative images of different coronal brain sections showing the immunostaining of Syt2 for PVcre (A) and PV ${ }^{\text {cre }}$ Syt $2^{\text {fl }}$ (B) mice. (C and $\mathbf{D}$ ) Deleting Syt2 from mPFC PV+ neurons did not affect sIPSCs received by pyramidal neurons. (C) Top, differential interference contrast (DIC) and fluorescence images showing the expression of EGFP-Cre in the mPFC of an Syt2 ${ }^{\mathrm{fl} / \mathrm{fl}}$ mouse. Slice is arranged upright; bottom, example traces showing the sIPSCs recorded from mPFC pyramidal neurons with (GFP side) and without (non-GFP side) deletion of Syt2 from PV ${ }^{+}$neurons. (D) Summary graph of the sIPSC frequency (left) and amplitude (right) recorded from mPFC pyramidal neurons on GFP and non-GFP sides ( $n=10$ non-GFP, $n=10$ GFP for both frequency and amplitude). (E and F) Deleting Syt2 from mPFC PV+ neurons did not affect evoked IPSCs received by pyramidal neurons. (E) Top, DIC and fluorescence images showing the expression of Cre-dependent CHiEF-tdTomato in mPFC PV ${ }^{+}$neurons of a PV cre Syt2 ${ }^{\mathrm{fl}}$ mouse. Slice is arranged upright; bottom, example traces showing the 1-ms, 45-Hz blue laser-evoked IPSCs (shown as blue vertical bars) recorded from mPFC pyramidal neurons in a PV cre Syt2 ${ }^{\text {fl }}$ mouse and a control mouse. (F) Summary graph of $45-\mathrm{Hz}$ light-evoked IPSC amplitude (in response to the first 10 train stimuli) recorded from mPFC pyramidal neurons in control and PV cre Syt $2^{\mathrm{fl}}$ mice ( $n=9$ control, $n=7 \mathrm{PV}^{\text {cre }}$ Syt $2^{\mathrm{fl}}$ ). For $\mathbf{D}$ and $\mathbf{F}$, data are shown as means \pm SEM from at least 3 independent litters. Scale bars: $1 \mathrm{~mm}$ (A); $0.5 \mathrm{~mm}$ (C, top); 50 pA (C, vertical); $0.5 \mathrm{~S}$ (C, bottom horizontal); $0.5 \mathrm{~mm}$ (E, top); $0.5 \mathrm{nA}$ (E, vertical); $0.1 \mathrm{~s}$ (E, bottom horizontal). 

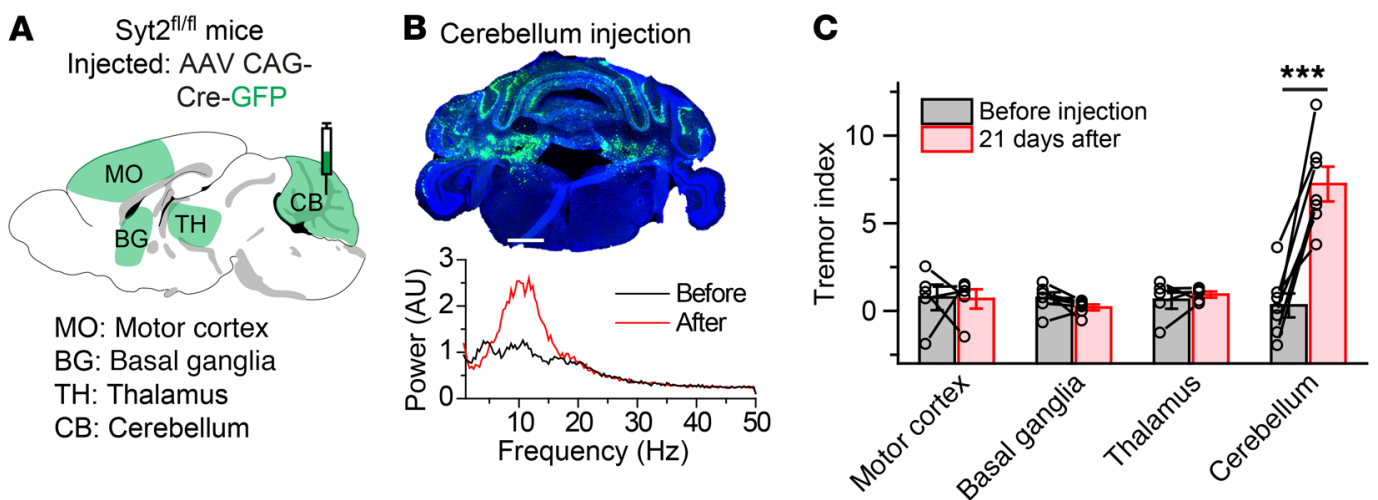

Figure 3. Syt2 deletion from the cerebellum is sufficient to generate an action tremor. (A) Stereotactic injection strategy of AAVs encoding Cre-GFP into 1 of 4 brain regions: the motor cortex (MO), basal ganglia (BC), thalamus (TH), or cerebellum (CB). (B) Top, a representative image showing the expression of Cre-GFP in the cerebellum of Syt2 ${ }^{\mathrm{fl} / \mathrm{fl}}$ mice; bottom, power spectrum of force-plate measurements from the same mouse before and after viral injection Also see Supplemental Video 5 and Supplemental Figure 5. (C) Summary graph of the tremor index before and after injections of AAV Cre-CFP into different brain regions ( $n=5 \mathrm{MO}, n=6 \mathrm{BG}, n=5 \mathrm{TH}, n=7 \mathrm{CB}$ ). Please see the methods section for our approaches to infect these large brain areas. For $\mathbf{C}$, data are shown as means \pm SEM from at least 3 independent litters. ${ }^{* *} P<0.001$ by 2 -sided, paired $t$ test. Scale bar: $1 \mathrm{~mm}$ (B).

(AAVs) expressing syn-EGFP-Cre unilaterally in the $\mathrm{mPFC}$ of Syt $2^{\mathrm{f} / \mathrm{l}}$ mice and recording sIPSCs from pyramidal neurons on either the GFP or the non-GFP side. Surprisingly, both the frequency and amplitude of recorded sIPSCs were normal on the GFP side, suggesting that Syt 2 knockout did not change sIPSCs in the cortex (Figure 2, C and D). We further recorded optogenetically evoked IPSCs by expressing DIO-CHiEF-tdTomato unilaterally in the $\mathrm{mPFC}$ of $\mathrm{PV}^{\text {cre }}$ Syt2 ${ }^{\mathrm{fl}}$ and control mice. In $\mathrm{PV}^{\text {cre }}$ Syt2 ${ }^{\mathrm{fl}}$ slices, $1-\mathrm{ms}, 45-\mathrm{Hz}$ laser-evoked IPSCs were also normal (Figure 2, E and F). These results suggest that synaptic release by cortical $\mathrm{PV}^{+}$neurons was not affected by the Syt 2 deletion, potentially due to the compensatory effect of synaptotagmin-1 (25), which could also explain the lack of other striking phenotypes besides tremor in PV ${ }^{\text {cre }}$ Syt2 ${ }^{\mathrm{fl}}$ mice.

Next, we employed a more direct approach to delete Syt 2 from different brain regions, and tried to identify the specific brain area in which the Syt 2 deletion is sufficient to generate an action tremor. We tested 4 candidate brain areas implicated in motor behaviors for this purpose: the motor cortex, basal ganglia, thalamus, and cerebellum. The motor cortex, including the primary and secondary motor cortex, provides command signals for voluntary movements. The basal ganglia are involved in movement initiation, and have been hypothesized to mediate the resting tremor that is a key symptom of Parkinson's disease. The thalamus includes relay centers for motor pathways, and the ventral intermediate nucleus of the thalamus is targeted by deep brain stimulation (DBS) to treat essential tremor (26-28). The cerebellum plays

Table 2. Cerebellar cell type expression patterns for $\mathbf{5}$ mouse Cre driver lines

\begin{tabular}{lcccc} 
Mouse line & Stellate cells & Basket cells & Purkinje cells & CBN cells \\
PV're & Yes & Yes & Yes & Yes \\
\hline L7 $^{\text {re }}$ & & & Yes & \\
Prkcd $^{\text {ree }}$ & Yes & Yes & Some & \\
Vglut2 $^{\text {cre }}$ & & & & Clutamatergic \\
Gad2 $^{\text {cre }}$ & Yes & Yes & Yes & CABAergic \\
\hline
\end{tabular}

a critical role in fine motor control, and pathological studies have detected cerebellar degeneration as a hallmark of essential tremor (5). Strikingly, removing Syt2 expression from the motor cortex, basal ganglia, or thalamus did not induce an action tremor (Supplemental Figure 5), whereas removing Syt2 from the cerebellum replicated the action tremor observed in $\mathrm{PV}^{\mathrm{cre}}$ Syt ${ }^{\mathrm{ft}}$ mice (Figure 3, A-C, Supplemental Figure 5E, and Supplemental Video 5). These results indicate that removing Syt 2 from neurons in the cerebellum was sufficient to generate an action tremor.

Syt2 deletion from $P V^{+}$neurons in the $C B N$ is sufficient to generate an action tremor. To pinpoint the cell types in the cerebellum that generate the action tremor in $\mathrm{PV}^{\mathrm{cre}}$ Syt $2^{\mathrm{fl}}$ mice, we took advantage of multiple Cre mouse lines that target different $\mathrm{PV}^{+}$cell types in the cerebellum (ref. 29, Table 2, and Supplemental Figure 6, A and B). We first crossed Syt $2^{\mathrm{t} / \mathrm{fl}}$ mice with $\mathrm{L}{ }^{\text {cre }}$ mice, which expressed Cre recombinase exclusively in Purkinje cells in the cerebellar cortex (Supplemental Figure 6, A and B). Degeneration of Purkinje cells has been hypothesized to be the cause of essential tremor (5). However, L $7^{\text {cre }}$ Syt $2^{\text {fl }}$ mice exhibited no action tremor (Figure 4 , A and C). Next, we crossed Syt $2^{\mathrm{f} / \mathrm{fl}}$ mice with Prkcd ${ }^{\text {cre }}$ mice (30), which expressed Cre recombinase in the molecular layer inhibitory neurons and in some Purkinje cells in the cerebellar cortex (ref. 31 and Supplemental Figure 6, A and B). Prkcd ${ }^{\text {cre }}$ Syt $2^{\text {fl }}$ mice were also normal and had no tremor phenotype (Figure 4C and Supplemental Figure 6C).

To test the roles of neurons in the CBN, we crossed Syt2 $2^{\mathrm{f} / \mathrm{fl}}$ mice with either Vglut2 ${ }^{\text {cre }}$ or Gad2 ${ }^{\text {cre }}$ mice, which expressed Cre recombinase in excitatory and inhibitory neurons, respectively (Supplemental Figure 6, A and B). Surprisingly, Vglut $2^{\text {cre }}$ Syt $2^{\mathrm{fl}}$ mice exhibited a robust tremor phenotype, whereas $\mathrm{Gad}^{\text {cre }}$ mice did not (Figure 4, B and C, and Supplemental Figure 6D). Besides the tremor phenotype, Vglut $2^{\text {cre }}$ Syt $2^{\mathrm{fl}}$ mice also had a significantly reduced body weight (Supplemental Figure 6E), presumably due to knockout of Syt2 from 
Cell type-specific Syt2 knockout in the cerebellum
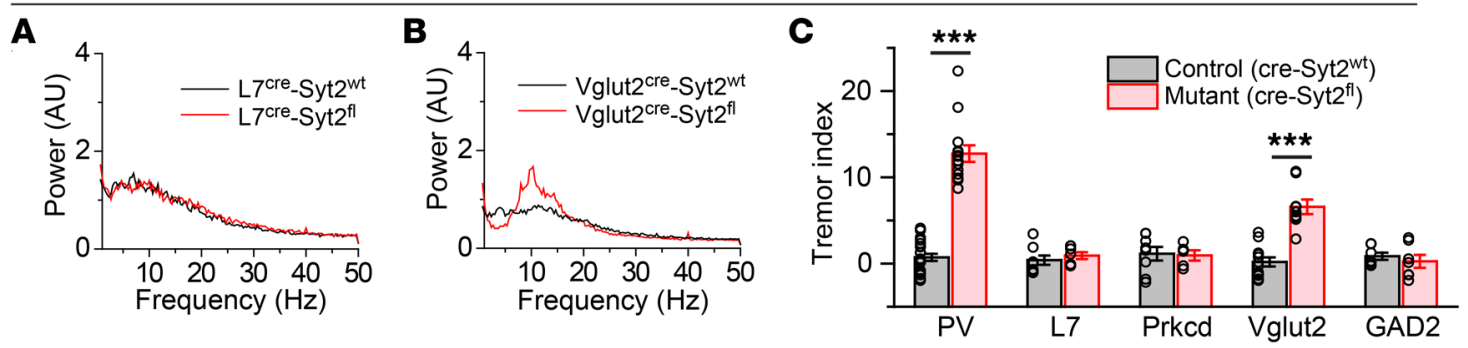

D
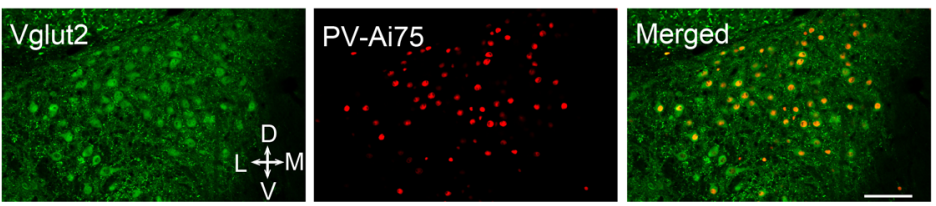

Syt2 knockout and rescue in the CBN

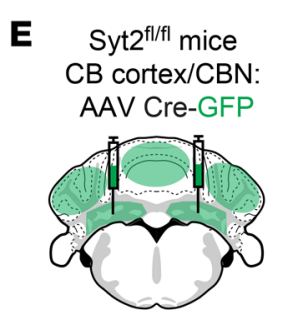

G
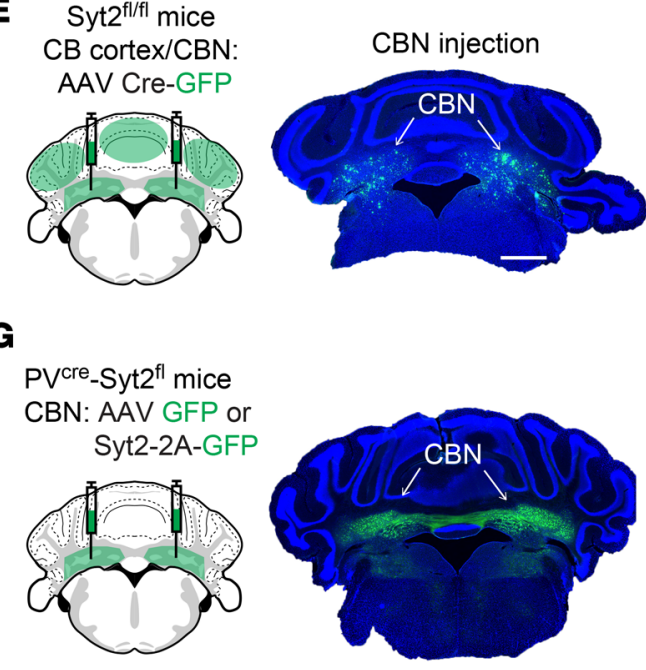
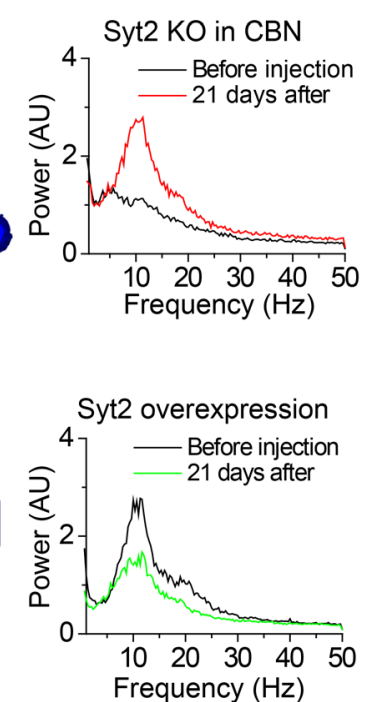

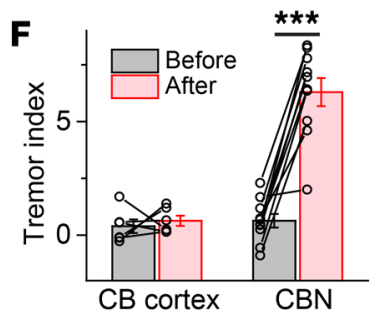

H

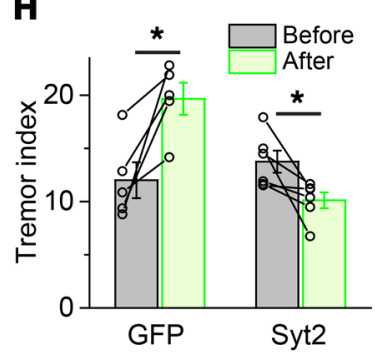

Figure 4. Syt2 deletion from $\mathbf{P V}^{+}$neurons in the CBN is sufficient to generate an action tremor. (A and B) Power spectra of force-plate measurements from representative control and $L 7^{\text {cre }}$ Syt2 $2^{\mathrm{fl}}$ mice (A) or control and Vglut2 ${ }^{\text {cre }}$ Syt2 $2^{\mathrm{fl}}$ mice (B). (C) Summary graph of the tremor index of Syt2 ${ }^{\mathrm{fl} / \mathrm{fl}}$ mice after crossing with the 5 different Cre mouse lines ( $n=19$ PV-control, $n=18$ PV-mutant, $n=8$ L7-control, $n=7$ L7-mutant, $n=8$ Prkcd-control, $n=5$ Prkcdmutant, $n=12$ Vglut2-control, $n=9$ Vglut2-mutant, $n=7$ GAD2-control, $n=7$ GAD2-mutant). (D) Representative images of the CBN section from a PVcre Ai75 mouse showing the colocalization of antibody-labeled Vglut2 neurons and genetically labeled $\mathrm{PV}^{+}$neurons in the CBN. (E) Left, stereotactic injection strategy of AAVs encoding Cre-GFP into the CBN or the cerebellar cortex of Syt2 ${ }^{\mathrm{fl} / \mathrm{fl}}$ mice; middle, a representative image showing the bilateral expression of Cre-GFP in the CBN; right, power spectrum of force-plate measurements from the same Syt2 $2^{\mathrm{fl} / \mathrm{fl}}$ mouse before and after Cre-CFP expression in the CBN. (F) Summary graph of tremor index before and after Cre-GFP expression in the CBN or cerebellar cortex of Syt2 $2^{\mathrm{fl} / \mathrm{fl}}$ mice $(n=6 \mathrm{CB}$ cortex, $n=10 \mathrm{CBN})$. (G and H) Figure panels arranged the same way as in $\mathbf{E}$ and $\mathbf{F}$, except that AAVs encoding GFP or Syt2-2A-GFP were injected into the CBN of PVcre Syt2 ${ }^{\mathrm{fl}}$ mice $(n=5$ GFP, $n=6$ Syt2). For $\mathbf{C}, \mathbf{F}$, and $\mathbf{H}$, data are shown as means \pm SEM from at least 3 independent litters. ${ }^{*} P<0.05 ;{ }^{* *} P<0.001$ by 2 -sided, unpaired $t$ test (C) or 2-sided, paired $t$ test (F and $\mathbf{H})$. Scale bars: $100 \mu \mathrm{m}(\mathbf{D}) ; 1 \mathrm{~mm}(\mathbf{E})$.

Vglut $2^{+}$excitatory neurons in brain regions other than the $\mathrm{CBN}$ that were not targeted by the PV ${ }^{\text {cre }}$ driver line. Since at least in cortical areas, $\mathrm{PV}^{+}$neurons are primarily inhibitory (32), we further crossed $\mathrm{PV}^{\text {cre }}$ mice with a nucleus-localized tdTomato reporter line (Ai75) and immunostained for Vglut2 in the CBN. Indeed, the majority of $\mathrm{PV}^{+}$neurons in the $\mathrm{CBN}$ colocalized with Vglut2 ${ }^{+}$ neurons, indicating that $\mathrm{PV}^{+}$neurons in the $\mathrm{CBN}$ were mostly glutamatergic (Figure 4D).

Both PV ${ }^{\text {cre }}$ and Vglut2 $2^{\text {cre }}$ mice expressed Cre in many other brain regions outside the cerebellum. Therefore, we further tested whether removing Syt2 from the CBN alone is sufficient to gener- ate an action tremor. Indeed, bilaterally injecting AAVs expressing Cre into the $\mathrm{CBN}$ of Syt $2^{\mathrm{f} / \mathrm{fl}}$ mice induced an action tremor with the same frequency range as observed in $\mathrm{PV}^{\text {cre }}$ Syt $2^{\mathrm{fl}}$ mice (Figure $4, \mathrm{E}$ and F), whereas expressing Cre in either the medial or lateral cerebellar cortex of Syt $2^{\mathrm{f} / \mathrm{l}}$ mice failed to replicate the tremor (Figure $4 \mathrm{~F}$ and Supplemental Figure 6, F and G). Furthermore, the agedependent tremor progression of $\mathrm{PV}^{\text {cre }}$ Syt $2^{\mathrm{tl}}$ mice was significantly rescued by overexpression of Syt 2 in the CBN (Figure 4, G and $\mathrm{H}$, and Supplemental Figure 6, H and I). Together, these results indicate that the deletion of Syt2 from the CBN was sufficient to generate an action tremor. 
A PVcre mice CBN: AAV DIO-mCherry
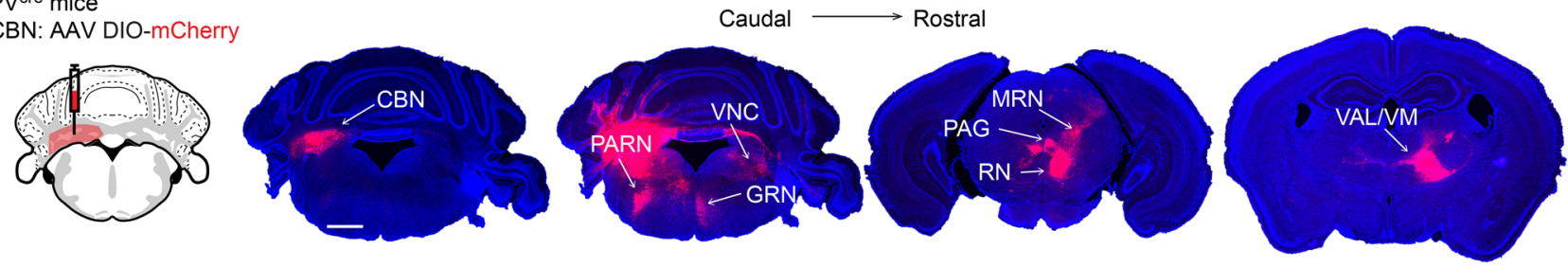

B
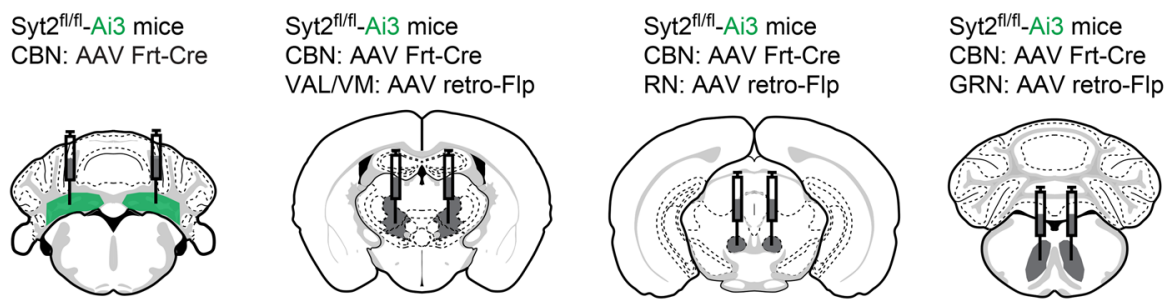

CBN: cerebellar nuclei

GRN: gigantocellular reticular nucleus CBN: AAV Frt-Cre

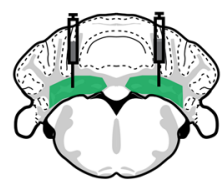

PARN: parvicellular reticular nucleus

VNC: vestibular nuclei

PAG: periaqueductal gray

MRN: midbrain reticular nucleus

$\mathrm{RN}$ : red nucleus

VAL: ventral anterior-lateral

complex of the thalamus

VM: ventral medial nucleus

of the thalamus

C

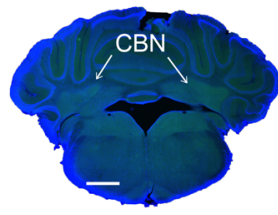

D

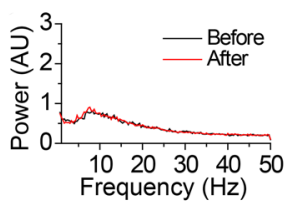

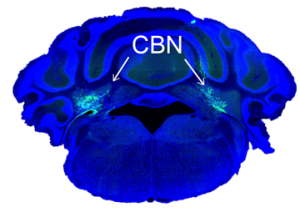

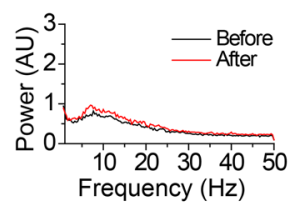

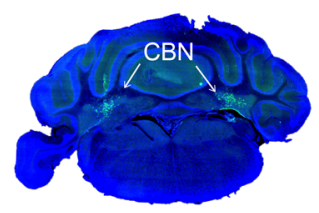
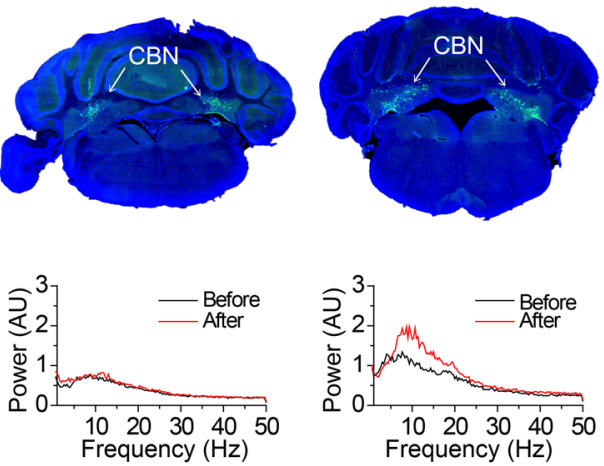

$\mathbf{E}$

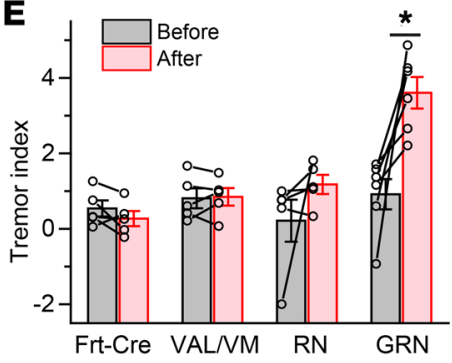

Figure 5. Identification of CBN downstream targets that induce the action tremor. (A) Representative images of anterograde tracing experiments of projections from $\mathrm{PV}^{+}$neurons in the CBN to other brain regions (left, stereotactic injection strategy of AAVs encoding DIO-mCherry into the CBN of PVcre mice; right images, coronal slices arranged in a caudal $\rightarrow$ rostral direction showing mCherry expression in the indicated brain regions [note that the 3 fluorescence images on the right were overexposed to reveal the axon terminal signals]). (B) Stereotactic injection strategies of AAVs encoding flippase-dependent Cre (Frt-Cre) in the CBN (most left) or both Frt-Cre in the CBN and AAV retro-flippase in the VAL/VM, red nucleus, or GRN of Syt2 $2^{f / f l}$ crossed with EYFP reporter (Syt2 Ai3) mice (3 on the right). (C) Representative images showing the expression of EYFP in CBN for each injection experiment depicted in B. (D) Power spectra of force-plate measurements from representative Syt2 Ai3 mice before and after the corresponding injections shown in B and C. (E) Summary graph of the tremor index before and after each injection shown in B $(n=5$ Frt-Cre, $n=5$ VAL/VM, $n=6$ RN, $n=5$ CRN). For $\mathbf{E}$, data are shown as means \pm SEM from at least 3 independent litters. ${ }^{*} P<0.05$ by Mann-Whitney test (E). Scale bars: $1 \mathrm{~mm}(\mathbf{A}) ; 1 \mathrm{~mm}(\mathbf{C})$.

Loss of fast synchronous neurotransmitter release at CBN $\rightarrow$ GRN synapses may induce the action tremor. To identify the downstream targets of $\mathrm{PV}^{+}$neurons in the $\mathrm{CBN}$ that generate the action tremor, we injected AAVs expressing Cre-dependent mCherry (DIO-mCherry) into the CBN of PV ${ }^{\text {cre }}$ mice. Three weeks later, robust mCherry signals were observed in the thalamus (ventral anterior-lateral complex of the thalamus, VAL; ventral medial nucleus of the thalamus, VM), the midbrain (red nucleus; midbrain reticular nucleus; periaqueductal gray), and the brainstem (gigantocellular reticular nucleus, GRN; parvicellular reticular nucleus; vestibular nuclei; Figure 5A). Among these brain regions, the VAL and VM may be analogous to the human ventral intermediate nucleus of the thalamus, which has been targeted for DBS treatment (26-28). The red nucleus and VAL were previously proposed to constitute an "essential tremor pathway" outside of the cerebellum (12). Moreover, the GRN has been shown to directly innervate motor neurons in the spinal cord, and therefore is in a good position to control fine motor movement $(33,34)$. We also performed more restricted injections of AAVs expressing DIO-mCherry into each individual nucleus of the CBN (fastigial nucleus; interposed nucleus; dentate nucleus; Supplemental Figure 7, A-C), and found that although all 3 nuclei projected to the brainstem, the midbrain, and the thalamus in general, there were some differences. For example, mainly the fastigial nucleus and dentate nucleus projected to the GRN (Supplemental Figure 7, A-C). Using retrograde tracing, we further confirmed that GRN neurons received robust inputs from the fastigial nucleus and dentate nucleus (Supplemental Figure 7, D and E).

To determine whether the same group of CBN neurons project to the GRN and to more rostral brain structures (red nucleus and VAL/VM), we injected retro-AAVs encoding mCherry into the GRN and retro-AAVs encoding GFP into either the red nucleus or the VAL/VM (Supplemental Figure 8, A and C). Subsequent imaging of labeled CBN neurons suggested that distinct $\mathrm{CBN}$ neurons projected to the GRN and red nucleus or VAL/VM (Supplemental Figure 8, $\mathrm{B}$ and $\mathrm{D}$ ). To test the roles of different $\mathrm{CBN}$ projections in action tremor, we bilaterally injected AAVs expressing wheat germ agglutinin-conjugated Cre (WGA-Cre) into the VAL/VM, red nucleus, or GRN of Syt $2^{1 / / 1 /}$ mice that had been crossed with Cre-dependent EYFP reporter mice (Ai3; Supplemental Figure 8, E and F). In this 


\begin{abstract}
A Mice: PVcre-Syt2 ${ }^{\text {wt }}$ or PVcre-Syt $2^{\text {fl }}$ CBN: AAV ChR2(H134R)-EYFP Recording from GRN neurons
\end{abstract}
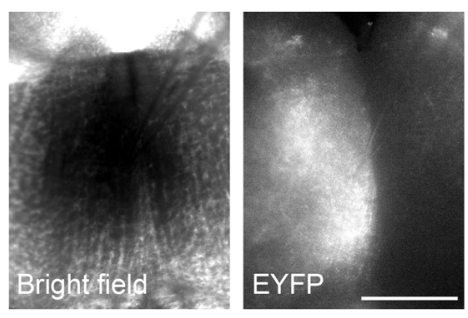

B

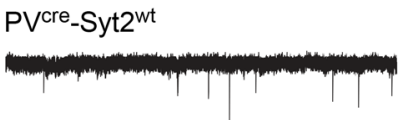

PVcre_Syt $2^{\text {fl }}$

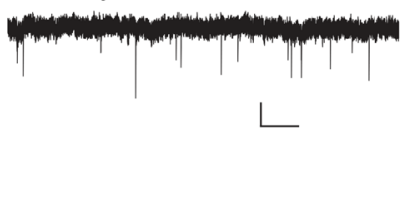

C

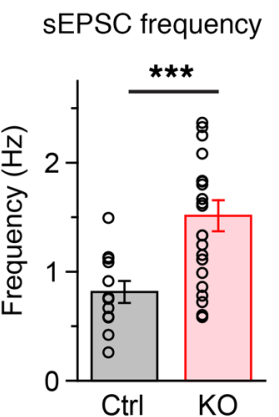

sEPSC amplitude

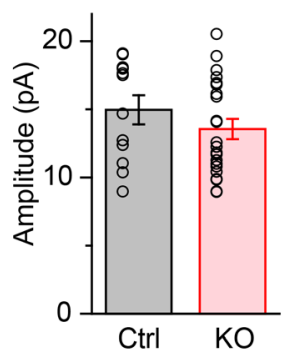

D L

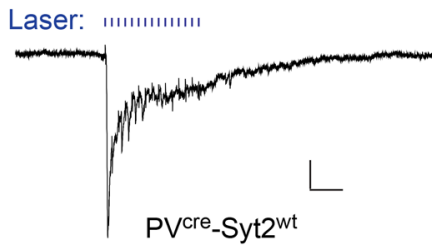

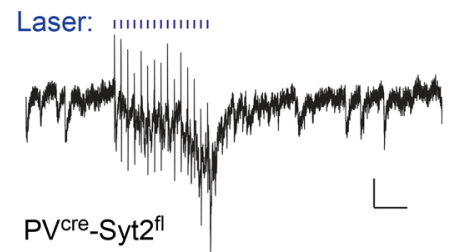

E

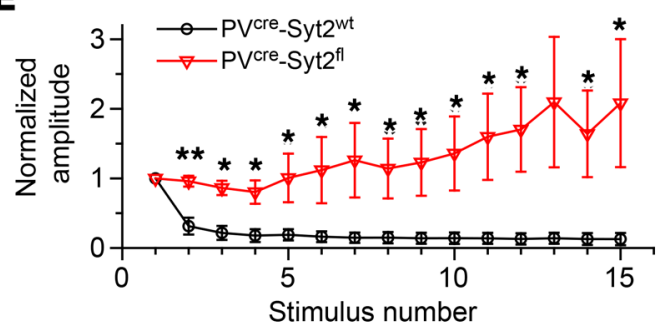

Figure 6. Loss of fast synchronous neurotransmitter release at CBN $\rightarrow$ GRN synapses may induce the action tremor. (A) Bright field and fluorescence images of GRN sections from PV cre Syt2 ${ }^{\mathrm{fl}}$ or control mice showing ChR2-EYFP expression in nerve terminals originating from CBN neurons. Slice is arranged upright. (B) Example traces showing spontaneous EPSCs recorded from GRN neurons in slices from control and PV cre Syt2 $2^{\text {fl }}$ mice. (C) Summary graph of the SEPSC frequency (left) and amplitude (right) recorded from GRN neurons in slices from control and PV ${ }^{\text {cre }}$ Syt $2^{f l}$ mice $\left(n=12\right.$ control, $n=22$ PV ${ }^{\text {cre }}$ Syt2 $2^{\text {fl }}$ for both frequency and amplitude). (D) Example traces showing 1-ms, 50- $\mathrm{Hz}$ blue laser-evoked EPSCs recorded from GRN neurons in control (left) and PVcre Syt2 ${ }^{\mathrm{fl}}$ (right) slices. (E) Summary of 50-Hz light-evoked EPSC amplitude recorded from GRN neurons in control and PV ${ }^{\text {cre }}$ Syt2 $2^{\mathrm{fl}}$ slices. All amplitudes are normalized to the first EPSC responses for both groups $\left(n=5\right.$ control, $n=7$ PVcre Syt $\left.2^{f 1}\right)$. For $\mathbf{C}$ and $\mathbf{E}$, data are shown as means \pm SEM from at least 3 independent litters. ${ }^{*} P<0.05$; ${ }^{* *} P<0.01 ;{ }^{* *} P<0.001$ by 2 -sided, unpaired $t$ test (C) or 2-sided, unpaired $t$ test (E). Scale bars: $0.5 \mathrm{~mm}$ (A); $10 \mathrm{pA}(\mathbf{B}$, vertical); $1 \mathrm{~s}$ (B, horizontal); 20 pA (D vertical left); $0.1 \mathrm{~s}$ (D horizontal left); 4 pA (D vertical right); $0.1 \mathrm{~s}$ (D horizontal right).

injection paradigm, WGA-Cre was retrogradely transported to presynaptic targets $(35,36)$, including the CBN, mediating the knockout of Syt2. We found that expression of WGA-Cre in the VAL/VM and red nucleus did not induce tremor (Supplemental Figure 8, E-I). Expression of WGA-Cre in the GRN severely impaired mice, necessitating euthanasia after about 1 week and preventing analysis of their tremor phenotype. This issue was most likely due to the WGACre-induced deletion of Syt2 in the numerous brainstem neurons that projected to the GRN (Supplemental Figure 7E), as suggested by the substantial endogenous Syt2 expression in the brainstem and the early lethality of constitutive Syt2 knockout mice (19).

To overcome this limitation and test the roles of CBN neurons projecting to the GRN, we adapted an intersectional method that deleted Syt2 in a pathway-specific manner (Figure 5B). Specifically, we used Syt2 ${ }^{\text {I//1 }}$ Ai3 mice to inject retro-AAVs encoding flippase into each of the 3 downstream candidate target nuclei, including the GRN, and regular AAVs expressing flippasedependent Cre recombinase (Frt-Cre) into the CBN (Figure 5, B and C). The results showed that removing Syt2 only from CBN neurons projecting to the GRN was sufficient to generate an action tremor (Figure 5, B-E).

To further identify the changes in synaptic neurotransmitter release produced by the knockout of Syt2, we expressed channelrhodopsin in the CBN of $\mathrm{PV}^{\text {cre }} \mathrm{Syt}^{\mathrm{fl}}$ or $\mathrm{PV}^{\text {cre }}$ Syt2 ${ }^{\text {wt }}$ mice and recorded synaptic responses from GRN neurons in acute slices using whole-cell voltage-clamp recordings (Figure $6 \mathrm{~A}$ ). We found that in $\mathrm{PV}^{\mathrm{cre}}$ Syt $2^{\mathrm{t}}$ slices, the frequency of spontaneous excitatory postsynaptic currents (sEPSCs), which are largely equivalent to miniature EPSCs (mEPSCs), was significantly increased, whereas the amplitude of sEPSCs was unchanged (Figure 6, B and C). Increases in $\mathrm{MEPSC}$ frequency are a typical phenotype induced by deletion of fast synaptotagmins (21). We next stimulated synaptic inputs to the GRN using blue laser light at $50 \mathrm{~Hz}$, and observed that fast synchronous neurotransmitter release was abolished in some of the GRN neurons recorded, whereas asynchronous neurotransmitter release appeared to be enhanced (Figure 6, D and E, and Supplemental Figure 8J). Moreover, we applied rabies tracing to label CBN neurons projecting to the GRN. Imaging results suggested that there were no obvious morphological differences between CBN neurons in PV ${ }^{\text {cre }}$ Syt $2^{\mathrm{t}}$ and $\mathrm{PV}^{\mathrm{cre}}$ Syt $2^{\mathrm{wt}}$ mice (Supplemental Figure 8, K-M). Together, these results suggest that the deletion of Syt2 from CBN neurons caused a loss of fast synchronous but not slower forms of neurotransmitter release, suggesting that a shift in the pattern of evoked neurotransmitter release in CBN $\rightarrow$ GRN synapses may be the cause of the action tremor observed in PV ${ }^{\text {cre }}$ Syt $2^{\mathrm{t}}$ mice.

Blocking neurotransmitter release in CBN neurons rescues the action tremor of $P V^{\text {ree }} S y t 2^{f l}$ mice and of harmaline-injected mice. To test whether the remaining asynchronous neurotransmitter release at $\mathrm{CBN} \rightarrow \mathrm{GRN}$ synapses in $\mathrm{PV}^{\text {cre }}$ Syt $2^{\mathrm{A}}$ mice induces the action tremor, we expressed Cre-dependent tetanus toxin (DIO-TetTox) in CBN neurons of PV ${ }^{\text {cre }}$ Syt2 ${ }^{\text {fl }}$ mice, which will block all synaptic release from $\mathrm{PV}^{+}$neurons in the $\mathrm{CBN}$ (ref. 37 and Figure 7A). Surprisingly, TetTox expression rescued instead 

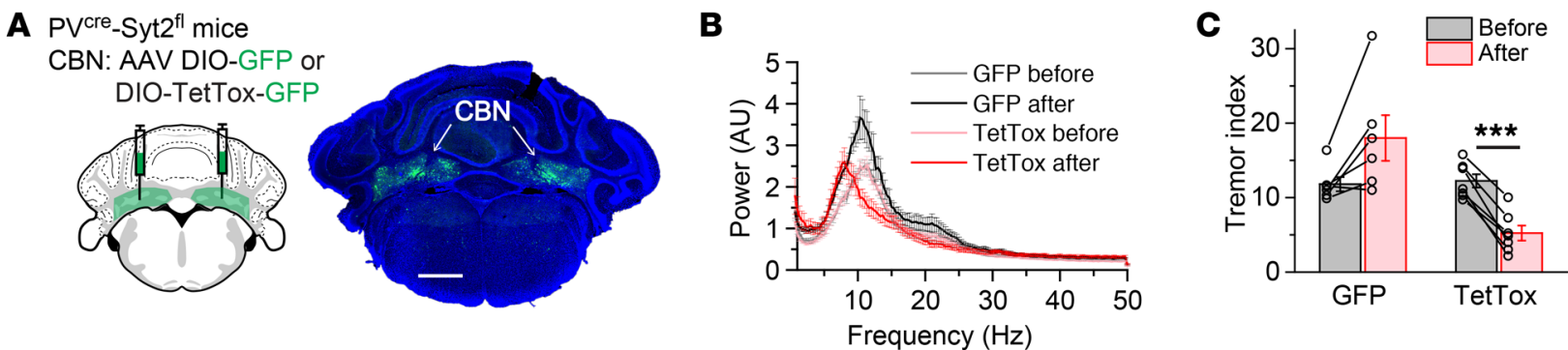

Figure 7. Blocking neurotransmitter release in CBN neurons rescues the action tremor of PVcre Syt ${ }^{\mathrm{fl}}$ mice. (A) Left, stereotactic injection strategy of AAVs

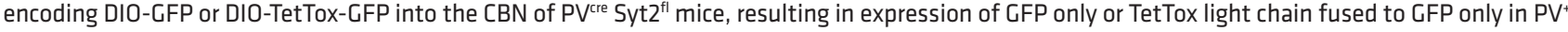
neurons; right, a representative image showing the bilateral expression of GFP in the CBN. (B) Averaged power spectrum of force-plate measurements from $\mathrm{PV}^{\text {cre }}$ Syt2 $2^{\mathrm{fl}}$ mice before and after injection of AAVs encoding GFP or TetTox-GFP ( $n=6$ GFP, $n=7$ TetTox). Note that after TetTox expression, the tremor not only decreased, but the peak tremor frequency also shifted to approximately $8 \mathrm{~Hz}$. (C) Summary graph of the tremor index before and after injection of AAVs encoding GFP or TetTox-GFP in the CBN of PV cre Syt2 ${ }^{f l}$ mice ( $n=6$ GFP, $n=7$ TetTox). For $\mathbf{B}$ and $\mathbf{C}$, data are shown as means \pm SEM from at least 2 independent litters. ${ }^{* *} P<0.001$ by 2 -sided, paired $t$ test. Scale bar: $1 \mathrm{~mm}(\mathbf{A})$.

of aggravated the age-dependent action tremor in $\mathrm{PV}^{\text {cre }}$ Syt2 ${ }^{\mathrm{t}}$ mice (Figure 7, B and C, and Supplemental Figure 9, A and B), suggesting that the action tremor of $\mathrm{PV}^{\text {cre }}$ Syt $2^{\mathrm{fl}}$ mice was generated by the malfunction of CBN $\rightarrow$ GRN synapses, instead of a simple loss of function at these synapses. A detailed spectral analysis showed that after TetTox expression, the $9-\mathrm{Hz}$ to $12-\mathrm{Hz}$ action tremor was largely gone, whereas an approximately $8 \mathrm{~Hz}$ tremor became manifest (Figure 7B and Supplemental Figure 9C). This TetTox-induced lower frequency tremor could be explained by 2 possible mechanisms, a frequency shift of the original $9-\mathrm{Hz}$ to $12-\mathrm{Hz}$ action tremor, or an approximately $8-\mathrm{Hz}$ tremor generated de novo. To understand the nature of this approximately 8-Hz tremor, we expressed DIO-TetTox in the CBN of PV ${ }^{\text {cre }}$ mice (Supplemental Figure 9D), which did not exhibit visible tremor. Interestingly, completely blocking the synaptic release in $\mathrm{CBN}$ $\mathrm{PV}^{+}$neurons in $\mathrm{PV}^{\text {cre }}$ mice induced tremor at approximately $8 \mathrm{~Hz}$ (Supplemental Figure 9, E and F), the same frequency range as the physiological tremor in $\mathrm{PV}^{\text {cre }}$ mice (Supplemental Figure 9, $\mathrm{E}$ and $\mathrm{G})$. This result suggests that the remaining approximately 8-Hz tremor after blocking all synaptic release from $\mathrm{PV}^{+}$neurons in the CBN of $\mathrm{PV}^{\text {cre }}$ Syt ${ }^{\mathrm{fl}}$ mice was not due to a frequency shift of the $9-\mathrm{Hz}$ to $12-\mathrm{Hz}$ action tremor.

Next, we tested whether completely blocking synaptic transmission in $\mathrm{CBN} \mathrm{PV}^{+}$neurons (Figure $8 \mathrm{~A}$ ) could also rescue the tremor phenotype in the harmaline-induced essential tremor model, which exhibited an $11-\mathrm{Hz}$ to $14-\mathrm{Hz}$ action tremor (Figure 8B and Supplemental Figure 10, A and B). Indeed, TetToxinjected $\mathrm{PV}^{\text {cre }}$ mice failed to exhibit the typical $11-\mathrm{Hz}$ to $14-\mathrm{Hz}$ action tremor normally induced by harmaline injections (Figure 8, C and D), although a reduction of locomotion partially contributed to this phenotype (Supplemental Figure 10C). Furthermore, we selectively blocked synaptic transmission in $\mathrm{CBN}$ $\mathrm{PV}^{+}$neurons that projected to the GRN or VAL/VM (Figure 8, $\mathrm{E}$ and $\mathrm{F}$ ). The harmaline-induced $11-\mathrm{Hz}$ to $14-\mathrm{Hz}$ tremor was completely eliminated after blocking the $\mathrm{CBN} \rightarrow$ GRN pathway, but not after blocking the $\mathrm{CBN} \rightarrow \mathrm{VAL} / \mathrm{VM}$ pathway (Figure 8 , G-I). Again, the reduction of locomotion partially contributed to these reductions in harmaline-induced action tremor (Supplemental Figure 10, D-F). Together, these results suggest that an increase in asynchronous neurotransmitter release at CBN
GRN synapses may be a general mechanism of action tremors observed in different animal models.

\section{Discussion}

In this study, we generated a mouse model that exhibited an action tremor with properties resembling the action tremor observed in human essential tremor disorder, suggesting that the mouse model we describe might help to gain insight into the etiology of the human essential tremor. There are 3 criteria for a valid animal disease model: face validity, predictive validity, and target validity (38). We found that $\mathrm{PV}^{\text {cre }}$ Syt $2^{\mathrm{fl}}$ mice exhibited a $9-\mathrm{Hz}$ to $12-\mathrm{Hz}$ action tremor that became progressively worse with age, replicating the major symptoms of essential tremor patients (face validity). The action tremor of $\mathrm{PV}^{\text {cre }}$ Syt $2^{\mathrm{fl}}$ mice was temporarily suppressed by ethanol, similar to the effect of alcohol in essential tremor patients (predictive validity). Moreover, we identified synapses formed by neurons in the CBN on GRN neurons in the brainstem as the cause of the action tremor in $\mathrm{PV}^{\mathrm{cre}}$ Syt $2^{\mathrm{fl}}$ mice, coinciding with the cerebellum's proposed tremorgenic role in human essential tremor patients (target validity). Together, these data suggest that $\mathrm{PV}^{\text {cre }}$ Syt $2^{\mathrm{fl}}$ mice have substantially added to existing animal models (14-18) to study the pathophysiology of essential tremor and to generate new ideas for therapeutic intervention.

Taking advantage of our $\mathrm{PV}^{\mathrm{cre}}$ Syt ${ }^{\mathrm{fl}}$ mouse model, we observed that dysfunction of excitatory $\mathrm{PV}^{+}$neurons in the CBN was sufficient to generate the action tremor. Previously, GABAergic Purkinje cells in the cerebellar cortex were proposed to play a critical role in essential tremor (5). Since glutamatergic neurons in the CBN are the major output neurons of the cerebellum and receive direct inputs from Purkinje cells, our results thus may have identified a key circuit component of essential tremor. In fact, some previous studies in humans and monkeys have suggested a role for the CBN in essential tremor (39-41).

Using intersectional genetic manipulation, we also demonstrated that the projection from the CBN to the GRN in the brainstem, but not the projection to the thalamus, was critical for generating the action tremor. Traditionally, the $\mathrm{CBN} \rightarrow$ thalamus $\rightarrow$ cortex pathway was proposed to be the "tremor pathway" (12), based on the finding that DBS of the ventral intermediate nucleus of the thalamus could suppress essential tremor in patients (26- 
DIO-TetTox expression in CBN of PVcre mice

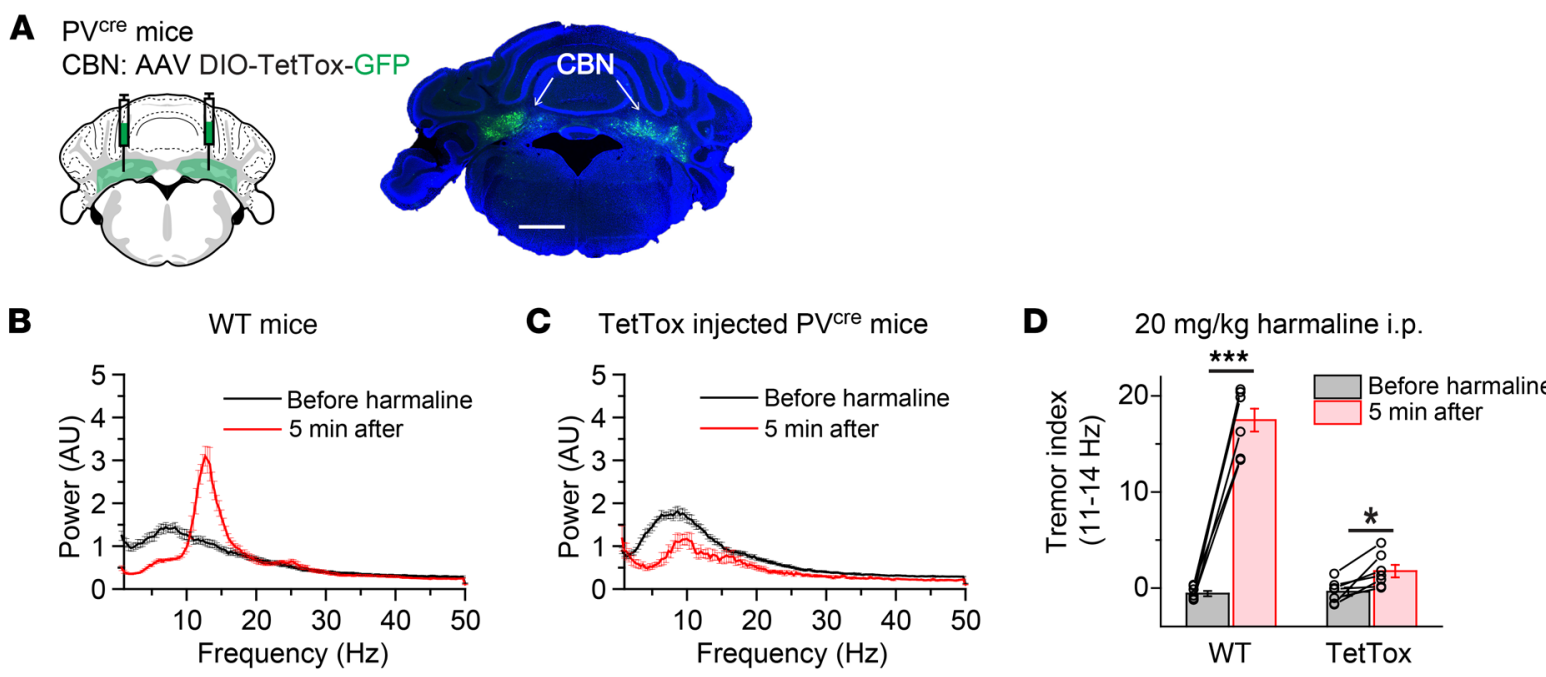

DIO-TetTox expression in CBN, Retro-Cre expression in GRN or VAL/VM of WT mice

$\mathbf{E}$

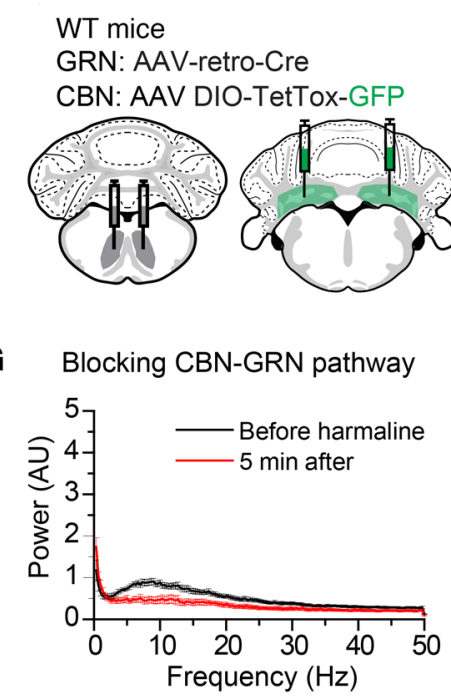

F WT mice

VAL/VM: AAV-retro-Cre

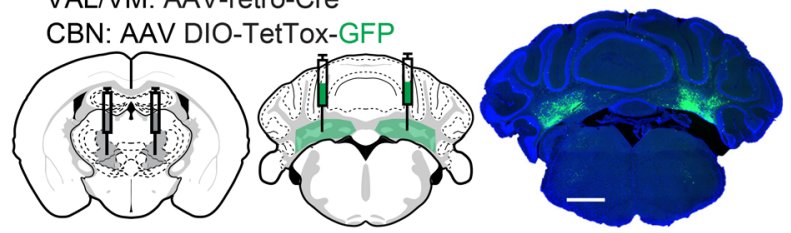

\begin{abstract}
H Blocking CBN-VAL/VM pathway
\end{abstract}

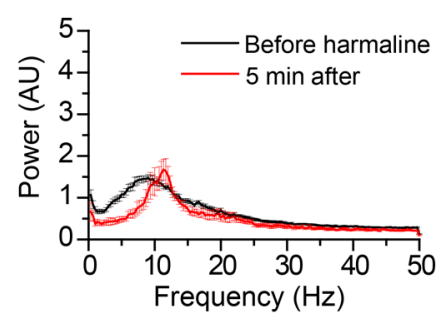

I

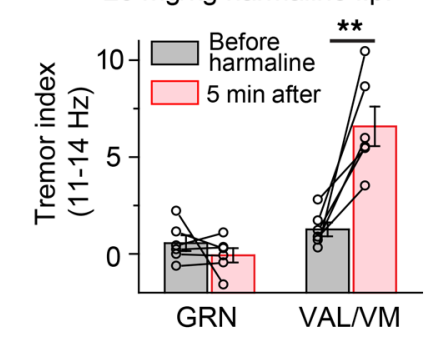

Figure 8. Blocking neurotransmitter release in CBN neurons rescues the action tremor of harmaline-injected mice. (A) Left, stereotactic injection strategy of AAVs encoding DIO-TetTox-GFP into the CBN of PV cre mice; right, a representative image showing the bilateral expression of GFP in the CBN. (B) Averaged power spectrum of force-plate measurements from WT mice before and 5 minutes after harmaline injection $(n=7)$. (C) Averaged power spectrum of force-plate measurements from TetTox-injected mice before and 5 minutes after harmaline injection $(n=7)$. (D) Summary of tremor index in the 11- $\mathrm{Hz}$ to 14- $\mathrm{Hz}$ range (see methods) before and 5 minutes after harmaline i.p. injection in WT and TetTox-injected mice $(n=7$ WT, $n=7$ TetTox). (E) Left and middle, stereotactic injection strategy of AAV-retro-Cre into the GRN and AAV DIO-TetTox-GFP into the CBN of WT mice; right, a representative image showing the bilateral expression of GFP in the CBN. (F) The same as in E, except that the AAV-retro-Cre virus was injected into the VAL/VM. (G) Averaged power spectrum of force-plate measurements from GRN-injected mice before and 5 minutes after harmaline injection $(n=6)$. (H) Averaged power spectrum of forceplate measurements from VAL/VM-injected mice before and 5 minutes after harmaline injection $(n=6)$. (I) Summary of tremor index in the $11-\mathrm{Hz}$ to $14-\mathrm{Hz}$ range before and 5 minutes after harmaline i.p. injection in GRN- and VAL/VM-injected mice ( $n=6 \mathrm{GRN}, n=6$ VAL/VM). For B-D and G-I, data are shown as means \pm SEM from at least 2 independent litters. ${ }^{*} P<0.05 ;{ }^{* *} P<0.01 ;{ }^{* *} P<0.001$ by 2 -sided, paired $t$ test. Scale bars: $1 \mathrm{~mm}(\mathbf{A}) ; 1 \mathrm{~mm}(\mathbf{E}) ; 1 \mathrm{~mm}(\mathbf{F})$.

28). There are 2 possible explanations for our findings. First, PV cre Syt $2^{\mathrm{fl}}$ mice may only recapitulate the mechanisms of a subgroup of essential tremor patients, for whom the disease mechanism is different from other essential tremor patients. Second, the major function of the $\mathrm{CBN} \rightarrow$ thalamus pathway may be to suppress instead of generate the action tremor. Our tracing results showed that neurons in the fastigial and dentate nuclei of the CBN projected to the GRN (Supplemental Figure 7, A-E), suggesting that these neurons were responsible for inducing the action tremor. Indeed, previous studies showed that cooling the dentate nucleus could induce an action tremor $(42,43)$. CBN neurons projecting to the red nucleus and VAL/VM were largely distinct from GRN-projecting neurons (Supplemental Figure 8, A-D). We speculate that these $\mathrm{CBN} \rightarrow$ thalamus projection neurons could be responsible for suppressing action tremor. From a clinical perspective, in the future it will be important to apply advanced neuroimaging techniques to record whether activities in the brainstem regions are correlated with the action tremor of essential tremor patients. It will also be 


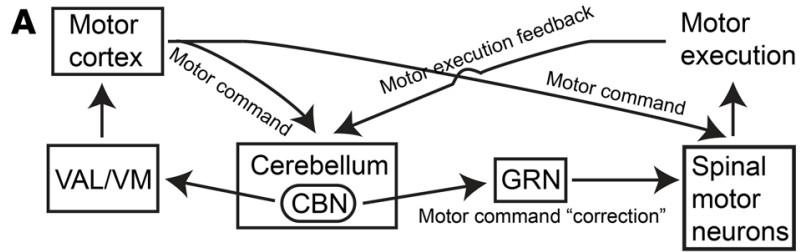

B

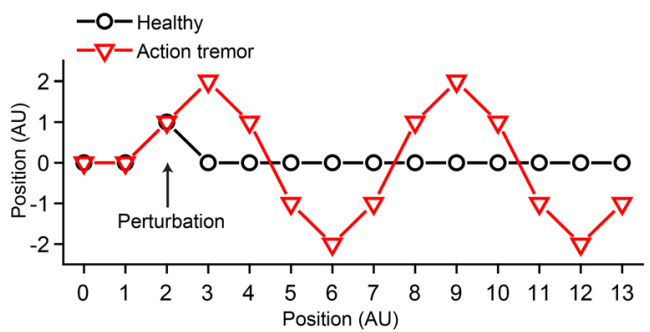

$\begin{array}{lllllllllllllll}\text { Real-time } Y \text {-axis } & 0 & 0 & -1 & 0 & 0 & 0 & 0 & 0 & 0 & 0 & 0 & 0 & 0 & 0\end{array}$

Figure 9. Model for the generation of action tremor. (A) Schematic diagram of the neural circuit controlling the real-time correction of movement command signals. (B) Simplified simulation showing how delayed online movement correction could generate oscillatory movement. Here the planned path is a straight line from $(0,0)$ to $(13,0)$; assuming movement at $(2,0)$ is deviated to $(2,1)$ due to a perturbation. In healthy animals, the movement is compensated by the $y$ axis "correction" signal "- 1 " in real time and continues its planned trajectory. In action tremor animals, we posit the correction signal is delayed by a fixed time period (time needed to move between 2 ticks on the $x$ axis), as shown by the row of numbers in red at the bottom. The deviated movement at $(2,1)$ is not compensated and continues on the wrong trajectory to $(3,2)$, where it starts to be partially compensated by the delayed correction signal -1 . Continuing with this delayed $y$ axis correction, the movement exhibits an oscillatory pattern. In this simplified simulation, as the duration of the fixed temporal delay increases, tremor amplitude increases and frequency decreases.

illuminating to test whether directly intervening in the CBN $\rightarrow$ GRN pathway (with DBS for example) would be a better treatment for essential tremor.

The synaptic deficits we observed at the $\mathrm{CBN} \rightarrow \mathrm{GRN}$ synapses (Figure 6, B-E) are consistent with previous studies of the role of synaptotagmins in clamping and mediating fast synchronous neurotransmitter release $(20,21,44,45)$. These synaptic changes correlate well with the tremor and provide interesting insights into the roles of the cerebellum in action tremor. In our working model (Figure 9A), we propose that the cerebellum receives a copy of the motor command and another copy of the motor execution feedback on a moment-by-moment basis. If these 2 signals are not perfectly matched, the cerebellar circuits will calculate the realtime "correction" signal needed to adjust and smoothen the ongoing movement (46). This hypothesized correction signal likely flows from the glutamatergic $\mathrm{PV}^{+}$neurons in the $\mathrm{CBN}$ to $\mathrm{GRN}$ neurons, which are directly connected to motor neurons in the spinal cord $(33,34)$, thereby adjusting the motor command signals in real time. If Syt2 is removed from glutamatergic neurons in the $\mathrm{CBN}$, this correction signal becomes temporally delayed and causes oscillations of the motor command signals, thereby inducing tremor (Figure 9B). Since this delayed correction signal only applies to the movement phase, the resulting tremor would be specifically an action tremor, as opposed to a resting tremor. Completely abolishing this pathologically delayed correction sig- nal, as by TetTox treatment, removed the oscillation of the motor command and blocked the $9-\mathrm{Hz}$ to $12-\mathrm{Hz}$ action tremor (Figure 9B). In general, our working model is consistent with the previous understanding of the role of the cerebellum in motor learning (47). Future studies will be needed to identify the neural circuits that transmit the motor command and execution feedback signals into the cerebellum, to characterize the local cerebellar circuit computing the correction signal, and to determine how oscillations are generated in downstream targets and how the frequency and amplitude of the resulting tremor are determined.

In this study, we used Syt2 as a molecular tool to identify the circuit components and synaptic defects underlying an action tremor. Given the wide distribution of $\mathrm{PV}^{+}$cells in the nervous system and the critical role of Syt2 as a fast $\mathrm{Ca}^{2+}$ sensor for neurotransmitter release $(19,20,45,48)$, it is somewhat unexpected that $\mathrm{PV}^{\text {cre }}$ Syt $2^{\mathrm{fl}}$ mice displayed a specific action tremor phenotype without exhibiting other dramatic behavioral defects (Supplemental Figure 3, B-E). We think there are 2 reasons for this phenotypic specificity. First, the function of Syt 2 could be compensated by other synaptotagmin isoforms that mediate fast and intermediate synchronous neurotransmitter release $(25,37)$, as supported by our recording results in the cortex (Figure 2, C-F). Second, many cells expressing Syt 2 as the only fast $\mathrm{Ca}^{2+}$ sensor are PV negative, as supported by the lethality in Syt 2 constitutive knockout mice (19) and the dramatic weight loss in Vglut $2^{\text {cre }}$ Syt2 ${ }^{\text {fl }}$ mice (Supplemental Figure 6E). Therefore, $\mathrm{PV}^{\mathrm{cre}}$ Syt $2^{\mathrm{fl}}$ mice serendipitously targeted, among others, a specific population of $\mathrm{PV}^{+}$neurons in the CBN (49) that use Syt2 as the only fast $\mathrm{Ca}^{2+}$ sensor and play critical roles in action tremor.

A limitation to our study is that Syt2 has not been shown as an essential tremor risk gene. Although the genetics of essential tremor are still poorly understood $(50,51)$, we do not think Syt2 will be identified as a risk gene for essential tremor patients, because of the lethality in Syt 2 constitutive knockout mice, which reveals its central importance in brain function. Nevertheless, we found that $\mathrm{PV}^{\text {cre }}$ Syt $2^{\mathrm{fl}}$ mice reproduced the major symptoms of essential tremor patients. The critical neural pathway and synaptic release defect we identified using this mouse model are likely downstream of the initial genetic cause and are probably shared by different animal models and human patients of essential tremor. Indeed, we found that blocking the abnormal neurotransmitter release in $\mathrm{CBN} \mathrm{PV}^{+}$neurons with TetTox could also rescue the action tremor of harmaline-injected mice (Figure 8, C and D). Our data thus suggest that $\mathrm{PV}^{\text {cre }}$ Syt $2^{\mathrm{fl}}$ mice are a reliable action tremor model that promises to be of use for studying the etiology of essential tremor and for developing therapeutic interventions in essential tremor patients.

\section{Methods}

Animals. Syt $2^{\mathrm{f} / \mathrm{fl}}$ mice were designed to have Syt 2 exon 2 flanked by loxP sites and were generated at the Janelia gene targeting and transgenics facility (21). They were backcrossed to C57BL/6J WT mice at least 7 times. C57BL/6J, PV ${ }^{\text {cre }}, \mathrm{L}^{\text {cre }}$, Vglut2 ${ }^{\text {cre }}, \mathrm{GAD}^{\text {cre }}, \mathrm{Ai3}$, Ai14, and Ai75 transgenic mice were purchased from The Jackson Laboratory. Prkcd ${ }^{\text {cre }}$ mice were provided by David Anderson's laboratory at The California Institute of Technology, Pasadena, California. Syt $2^{\mathrm{t} / \mathrm{fl}}$ mice were crossed with each different Cre driver line 2 generations to 
generate Driver ${ }^{\text {cre/cre }}$ Syt $2^{\mathrm{fl} /+}$ mice. These mice were then crossed with Syt $2^{\mathrm{fl} / \mathrm{fl}}$ mice to generate littermate control (Driver ${ }^{\mathrm{cre} /+}$ Syt $2^{\mathrm{fl} /+}$ ) and knockout mice (Driver ${ }^{\text {cre/+ }}$ Syt $2^{\mathrm{t} / \mathrm{fl}}$ ) for experiments. The littermate control mice were used in all related experiments except for results shown in Figure 2, A and B, and Supplemental Figure 4, in which $\mathrm{PV}^{\text {cre }}$ mice were used as the control. Genotyping was performed by Transnetyx. Mice were group housed (maximum 5 mice in a cage) on a 12-hour light/12-hour dark cycle (7 am to $7 \mathrm{pm}$, light) with food and water freely available. Roughly equal numbers of males and females were used for all experiments.

Vector construction and AAV preparation. AAV CAG-Cre-GFP and AAV CAG-GFP-2A Syt2 were packaged with AAV-DJ capsids for high efficiency in vivo neuronal infection. Virus was prepared with a procedure as previously described (36). Briefly, the CAG-Cre-GFP or AAV CAG-GFP-2A Syt2 constructs were cotransfected with pHelper and pRC-DJ into HEK293 cells (ATCC). Then, 72 hours later, cells were collected, lysed, and loaded onto iodixanol gradient for centrifugation at $80,000 \mathrm{~g}$ for 2 hours. The fraction with $40 \%$ iodixanol of the gradient was collected, washed, and concentrated with 100,000 MWCO tube filter. The genomic titer of virus was measured by quantitative PCR (qPCR).

Stereotaxic injection. AAV CAG-Cre-GFP and AAV CAG-GFP-2A Syt2 viruses were homemade. AAV retro-flippase, AAV Syn-Frt-CreIRES-tdTomato, and rabies viruses were produced by the Janelia viral tools facility. AAV-retro-BFP-Cre was produced by Addgene. All the other AAVs were produced by the Stanford Virus Core. They were all in AAV-DJ serotype unless noted otherwise. The concentrations of virus used for stereotaxic injection were adjusted to $1.0 \times 10^{12}$ genomic units/mL. Mice 45 to 60 days old ( 21 days for electrophysiological recording experiments) were anesthetized with tribromoethanol $(300 \mathrm{mg} / \mathrm{kg}$ ) and head-fixed with a stereotaxic device (KOPF model 1900). Viruses were injected using a glass micropipette attached to a 10- $\mu$ L Hamilton syringe. The pipette tips were beveled to be sharp and smooth. AAVs were injected at a flow rate of $0.15 \mu \mathrm{L} / \mathrm{min}$ bilaterally for behavior experiments and on the left side for tracing experiments, unless otherwise noted. We waited for 2 minutes before and 4 minutes after each injection. AAVs were allowed to express for 3 weeks, after which mice were acutely sectioned for slice electrophysiology, histologically processed for anatomical tracing, or tested on the force plate and then histologically processed to confirm viral injection sites. For the rabies tracing experiments, $0.2 \mu \mathrm{L}$ of a 1:1 volume mixture of AAV5CAG-DIO-avian tumor virus receptor A-mCherry (TVA-mCherry) and AAV8-CAG-DIO-glycoprotein was injected into the left GRN of 6- to 8-week-old PV ${ }^{\text {cre }}$ Syt $2^{\text {fl }}$ or control mice (36). Two weeks later, 0.3 $\mu \mathrm{L}$ glycoprotein-deleted rabies virus was injected into the same brain region. One week later, mice were perfused and processed for imaging. The coordinates for small brain structures in millimeters were the following (anterior to bregma, lateral to midline, ventral to dura; volume): CBN $(-6.2,1.75,2.5 ; 0.2 \mu \mathrm{L}) ; \operatorname{VAL} / \mathrm{VM}(-1.55,1.1,4 ; 0.2 \mu \mathrm{L})$; red nucleus $(-3.38,0.75,4.3 ; 0.2 \mu \mathrm{L})$; GRN $(-6.8,0.4,5.15 \rightarrow 4.25 ; 0.5 \mu \mathrm{L})$; fastigial nucleus $(-6.55,1,2.5 ; 0.05 \mu \mathrm{L})$; interposed nucleus $(-6.2,1.75$, 2.5 ; $0.05 \mu \mathrm{L})$; dentate nucleus $(-6,2.2,2.5 ; 0.05 \mu \mathrm{L})$. For each large brain area, viruses were injected at multiple sites at $0.25 \mu \mathrm{L} /$ minute. The pipette tip was moved from the ventral coordinate to the dorsal coordinate during infusion at a steady speed in order to increase infection areas. Coordinates were the following: $\operatorname{mPFC}(1.25,0.3,1.4 \rightarrow$ $0.4 ; 1 \mu \mathrm{L})$; motor cortex $(1.8, \pm 1.25,0.9 \rightarrow 0.1 ; 1 \mu \mathrm{L}),(1.2, \pm 1.2,0.9 \rightarrow$
$0.1 ; 1 \mu \mathrm{L}),(0.6, \pm 1.1,0.9 \rightarrow 0.1 ; 1 \mu \mathrm{L})$, and $(-0.2, \pm 0.9,0.9 \rightarrow 0.1 ; 0.75$ $\mu \mathrm{L})$; basal ganglia $(0.4, \pm 2,3.6 \rightarrow 2 ; 2 \mu \mathrm{L}),(-0.2, \pm 2.2,3.6 \rightarrow 2 ; 2 \mu \mathrm{L})$, and $(-0.8, \pm 2.5,3.6 \rightarrow 2 ; 1 \mu \mathrm{L})$; thalamus $(-0.8, \pm 0.75,4 \rightarrow 3 ; 1 \mu \mathrm{L})$, $(-1.31, \pm 1,4.3 \rightarrow 3.3 ; 2 \mu \mathrm{L})$, and $(-1.9, \pm 1,4 \rightarrow 3 ; 2 \mu \mathrm{L})$; cerebellum $(-5.8$, $\pm 0.75,2.3 \rightarrow 0.7 ; 1.5 \mu \mathrm{L}),(-5.8, \pm 2.25,2.3 \rightarrow 0.7 ; 1.5 \mu \mathrm{L}),(-6.35,0,2.3$ $\rightarrow 0.7 ; 1.5 \mu \mathrm{L}),(-6.35, \pm 1.5,2.3 \rightarrow 0.7 ; 1.5 \mu \mathrm{L}),(-6.35, \pm 3,2.3 \rightarrow 0.7$, $1.5 \mu \mathrm{L}),(-7, \pm 0.75,2.1 \rightarrow 0.7 ; 1.2 \mu \mathrm{L})$, and $(-7, \pm 2.25,2.1 \rightarrow 0.7 ; 1.2 \mu \mathrm{L})$; lateral cerebellar cortex $(-5.8, \pm 2.75,1 ; 1 \mu \mathrm{L}),(-6.35, \pm 3,1 ; 1 \mu \mathrm{L})$ and $(-7$, $\pm 2.5,1 ; 1 \mu \mathrm{L})$; medial cerebellar cortex $(-5.8, \pm 0.5,1 ; 1 \mu \mathrm{L}),(-6.35, \pm 0.5$, $1 ; 1 \mu \mathrm{L})$, and $(-7, \pm 0.5,1 ; 1 \mu \mathrm{L})$.

Behavior. Male and female mice 65 to 80 days old were used for all behavior tests unless noted otherwise. The genetic background of the mice and type of virus injected were coded and blinded to the experimenters.

Force plate. The design and applications of the same force-plate actometer device were described in detail in a previous study (22). We took advantage of this device's high sampling rate $(100 \mathrm{~Hz})$ of weight measurement and used a custom MATLAB script for data analysis. Mice were individually placed on the $28 \times 28 \mathrm{~cm}$ plate and allowed to freely explore for 5 minutes unless noted otherwise. The raw data were divided into 3-second segments and fast Fourier transformation was performed for each 3-second segment. The power spectra were then averaged (Figure 1C). A "tremor index" was calculated by integrating the power value in the $9-\mathrm{Hz}$ to $12-\mathrm{Hz}$ window. Power value in the $3-\mathrm{Hz}$ to $6-\mathrm{Hz}$ window was used as the baseline. Real-time locomotion distance information could be derived from the same force-plate raw data using the Pascal programs that came with the force-plate device. For ethanol injection experiments, animals were first measured on the force plate for 5 minutes, then given s.c. injections of $2.5 \mathrm{~g} / \mathrm{kg}$ ethanol or saline and measured on the force plate for another 60 minutes. For harmaline injection experiments, $1 \mathrm{mg} / \mathrm{mL}$ harmaline solution was prepared fresh by dissolving harmaline hydrochloride (MilliporeSigma, H1392) in saline. Animals were first measured on the force plate for 5 minutes, then given i.p. injections of $20 \mathrm{mg} / \mathrm{kg}$ harmaline and measured on the force plate for another 20 minutes. The action tremor was induced approximately 5 minutes after harmaline injection. The 5- to 10-minute window was analyzed for harmaline-induced tremor.

Video analysis of tremor. To simultaneous collect video and forceplate data, mice were placed on the force plate and surrounded by a small $(13.35 \times 8.40 \times 20 \mathrm{~cm})$ custom floorless acrylic chamber (TAP Plastic) suspended over the force plate. Video recording was performed with an iPhone $\mathrm{X}$ mounted on top of the acrylic chamber using slow-motion mode (240 frames per second). Each behavioral session consisted of 2 minutes of free exploration, and the raw force-plate data and video were saved for offline analysis. Using a tracking software Viewer III (BIOBSERVE), we extracted the $x-y$ position of the animal's nose over time (Supplemental Figure 2, A and B). With a custom MATLAB script, these coordinates were bandpass filtered $(9-12 \mathrm{~Hz})$ to produce the filtered $x$ and filtered $y$ signals. The envelopes (instantaneous amplitude) of these 2 signals were then calculated using the Hilbert transform (Supplemental Figure 2C and Supplemental Videos 1 and 2). Instantaneous tremor amplitude was calculated by taking the Euclidean norm of individual $x$ and $y$ instantaneous amplitude. This was then averaged to calculate the average tremor amplitude for the session.

Spontaneous alternation Y maze. A light gray plastic Y maze was used to evaluate spatial working memory. The maze consisted of 3 arms separated by $120^{\circ}$ (dimensions of each arm: $40 \times 10 \times 17 \mathrm{~cm}$ ). 
Mice around 4 weeks old were individually placed in the distal end of one arm and allowed to freely explore the whole maze for 10 minutes. Completed arm entry was defined as the entering of a whole mouse including its tail into an arm. The sequences and total numbers of arm entries were recorded and analyzed with the Viewer III tracking system. Visiting all 3 different arms consecutively was termed a "correct" trial, and visiting one arm twice or more in 3 consecutive entries was termed "wrong" trial. We calculated the correct alternation percentage as (number of correct trials/total number of correct and wrong trials) $\times 100$.

Novel object recognition. The same boxes used for open field test were used. On day 1 , mice around 1 month old were given 10 minutes of habituation time individually in an empty box. On day 2, mice were individually placed in the box for 10 minutes with 2 identical objects, either T75 cell culture flask filled with bedding material or blocks of LEGOs. On day 3, one object was replaced with a novel object. Object location in chamber was randomized. Exploration behavior was recorded and analyzed with the Viewer III tracking system. The recognition index was defined as the time spent on the novel object (or the left object for training phase) divided by the time spent on both objects.

Rotarod. An accelerating rotarod designed for mice (IITC Life Science) was used. The test consisted of 3 trials per day over the course of 3 days. The rotarod was activated after placing mice on the motionless rod. The rod accelerated from 4 to 40 revolutions per minute in 5 minutes. Each trial ended when a mouse fell off, made 1 complete revolution while hanging on, or reached 300 seconds.

Fear conditioning. On training day, mice were individually placed in a fear-conditioning chamber (Coulbourn Instruments) located in the center of a sound-attenuating cubicle. The conditioning chamber was cleaned with $10 \%$ ethanol to provide a background odor. A ventilation fan provided a background noise at approximately $55 \mathrm{~dB}$. After a 2-minute exploration period, 3 tone-foot shock pairings separated by 1-minute intervals were delivered. The $85-\mathrm{dB}, 2-\mathrm{kHz}$ tone lasted for 30 seconds and the foot shock was $0.75 \mathrm{~mA}$ and lasted for 2 seconds. The foot shocks coterminated with the tone. The mice remained in the training chamber for another 60 seconds before being returned to home cages. In the context test, mice were placed back into the original conditioning chamber for 5 minutes. The behavior of the mice was recorded with Freezeframe software and analyzed with Freezeview software (Coulbourn Instruments). The running speed of the mice was analyzed offline with Viewer III software. The average running speeds of mice in the 2-minute exploration period and 1-minute period after each foot shock were summarized as an indication of contextual fear memory acquisition. The average running speed of mice in the 5minute period during the context recall test was summarized as an indication of contextual fear memory retrieval.

Acute brain slice electrophysiology. P21 mice were used for viral injections, and slice recordings were performed 20 to 30 days later. To increase visibility and obtain better recording quality of GRN neurons from adult brainstem slices, we slightly modified an $N$-methyl-D-glucamine-based (NMDG-based) protocol (52). Specifically, $160-\mu \mathrm{m}$ coronal brainstem slices were cut with a vibratome (VT1200S, Leica) in chilled cutting solution (in mM): 92 NMDG, 2.5 $\mathrm{KCl}, 1.25 \mathrm{NaH}_{2} \mathrm{PO}_{4}, 20$ HEPES, $10 \mathrm{MgSO}_{4}, 0.5 \mathrm{CaCl}_{2}, 30 \mathrm{NaHCO}_{3}, 25$ D-glucose, 2 thiourea, $5 \mathrm{Na}$-ascorbate, $3 \mathrm{Na}$-pyruvate ( $\mathrm{pH} 7.3$, adjusted with $\mathrm{HCl}$ ) saturated with $95 \% \mathrm{O}_{2} / 5 \% \mathrm{CO}_{2}$ and recovered in the same NMDG-based cutting solution at $33^{\circ} \mathrm{C}$ for 10 to 15 minutes. Slices were then transferred to room temperature oxygenated artificial cerebrospinal fluid (ACSF) containing (in $\mathrm{mM}$ ): $119 \mathrm{NaCl}, 2.5$ $\mathrm{KCl}, 1.25 \mathrm{NaH}_{2} \mathrm{PO}_{4}, 2 \mathrm{MgSO}_{4}, 2 \mathrm{CaCl}_{2}, 26 \mathrm{NaHCO}_{3}, 12.5$ D-glucose, 2 thiourea, $5 \mathrm{Na}$-ascorbate, $3 \mathrm{Na}$-pyruvate. After slices were transferred to a recording chamber, oxygenated ACSF was continuously perfused. Whole-cell voltage-clamp recordings were made with 3- to 4-M $\Omega$ pipettes filled with internal solution containing (in $\mathrm{mM}$ ): 140 $\mathrm{CsMeSO}_{4}, 2 \mathrm{CsCl}, 10$ HEPES, 10 EGTA, $0.3 \mathrm{Na}_{2}$-GTP, 2 Mg-ATP, 7 phosphocreatine, 5 TEA-Cl, 1 QX314 (pH 7.3, adjusted with $\mathrm{CsOH}$ ). The whole-cell current signals were recorded with MultiClamp 700B and Clampex 10.4 data acquisition software (Molecular Devices). Recordings were made from neurons in the GRN under bright field visualization with an upright microscope (BX51WI, Olympus). After establishment of the whole-cell configuration and equilibration of the intracellular pipette solution with the cytoplasm, sEPSCs were recorded by holding the cell at $-70 \mathrm{mV}$. Spontaneous EPSCs were recorded for 5 minutes. A 473-nm laser (OEM Laser Systems) was used for light-evoked EPSC recordings. Light was delivered through an optic fiber ( $200 \mu \mathrm{m}, 0.22 \mathrm{NA})$ pointing toward the recorded area. Laser intensity was adjusted to get a maximal response without overstimulation; $50-\mathrm{Hz}, 1-\mathrm{ms}$ blue light was applied in 3 repetitions at 20 -second intervals. Synaptic currents were analyzed offline using Clampfit 9 (Molecular Devices) software. Spontaneous events were analyzed using the template matching search and a minimum threshold of $5 \mathrm{pA}$, and each event was visually inspected by an experimenter blind to the experiment conditions. Slice recordings for $\mathrm{MPFC}$ neurons were performed similarly, except that a regular sucrosebased slice cutting protocol was used (35). sIPSCs were recorded by using a regular high-chloride internal solution (36), including $20 \mu \mathrm{M}$ 6-cyano-7-nitroquinoxaline-2,3-dione (CNQX), $50 \mu \mathrm{M}(2 R)$ amino-5-phosphonovaleric acid (APV) in the ACSF, and clamping the neurons at $-70 \mathrm{mV}$. Evoked IPSCs were recorded by using the same internal solution as GRN recordings, including $20 \mu \mathrm{M} \mathrm{CNQX}$, $50 \mu \mathrm{M}$ APV in the ACSF, and clamping the neurons at $0 \mathrm{mV}$.

Histology. Mice were deep anesthetized with tribromoethanol and perfused with $10 \mathrm{~mL}$ of PBS followed by $10 \mathrm{~mL}$ of fixative (4\% paraformaldehyde diluted in PBS). The brains were removed and postfixed in $4^{\circ} \mathrm{C}$ overnight, and then immersed in $30 \%$ sucrose solution for 2 days before being sectioned at $50-\mu \mathrm{m}$ thicknesses on a cryostat (Leica CM3050 S). The free-floating brain sections were collected in PBS. For injection site verification, the sections were directly mounted onto glass slides with Vectashield mounting medium with DAPI, except for results shown in Supplemental Figure 7D, in which mounting medium without DAPI was used. For tracing experiments, 1 out of every $5 \mathrm{sec}-$ tions was collected for the whole brain. For immunohistochemistry, standard procedures were followed (36). We used primary antibodies to Syt2 (rabbit, A320, 1:1000, ref. 19), Vglut1 (guinea pig, Millipore AB5905, 1:1000), and Vglut2 (guinea pig, Millipore AB2251, 1:1000). A scanning microscope (BX61VS, Olympus) was used to scan fluorescence images for whole brain slices, and a confocal microscope (Nikon A1) was used for higher resolution imaging.

Statistics. All results are presented as mean \pm SEM and were analyzed by OriginPro 8 software (OriginLab Corp.). No statistical methods were used to predetermine sample sizes, but our sample sizes were similar to those reported in previous publications. Normality tests and $F$ tests for equality of variance were performed before choosing the 
statistical test. Unless otherwise indicated, statistics were based on 2-sided unpaired or paired $t$ tests or Mann-Whitney tests (for data sets that were not normally distributed) for 2 -group comparisons. $P<0.05$ was considered significant $\left({ }^{*} P<0.05,{ }^{* *} P<0.01,{ }^{* * *} P<0.001\right)$. Results in Figure 1I were analyzed with 1-way repeated measures ANOVA with post hoc analysis (Tukey's). Whenever possible, experimenters were blinded to experiment conditions. Injection sites and viral expression were confirmed for all animals. Mice showing incorrect injection sites were excluded from data analysis.

Study approval. All animal experiments were conducted following protocols approved by the Administrative Panel on Laboratory Animal Care at Stanford University.

\section{Author contributions}

MZ, WX, and TCS designed the experiments. MZ, MDM, and WX performed the experiments. MZ and TCS analyzed the data and wrote the manuscript with input from all authors.

\section{Acknowledgments}

We thank V. Cheepurupalli, J. Vu, and M. Tran for technical assistance; D. Anderson for providing Prkcd ${ }^{\text {cre }}$ mice; H. Wu and C. Halpern for advice on clinical aspects of the project. The work was supported by an NIH/National Institute of Mental Health grant (MH052804 to TCS) and an NIH/National Institute of Neurological Disorders and Stroke grant (1K01NS105155 to MZ).

Address correspondence to: Mu Zhou, 265 Campus Drive, Stanford, California 94305, USA. Phone: 650.721.1421; Email: muuzhou@gmail.com.

MDM's present address is: UCLA-Caltech MSTP program, Los Angeles, California, USA.

WX's present address is: Department of Neuroscience, UT Southwestern Medical Center, Dallas, Texas, USA.
1. Louis ED, Ferreira JJ. How common is the most common adult movement disorder? Update on the worldwide prevalence of essential tremor. Mov Disord. 2010;25(5):534-541.

2. Haubenberger D, Hallett M. Essential tremor. N Engl J Med. 2018;378(19):1802-1810.

3. Dogu O, et al. Prevalence of essential tremor: door-to-door neurologic exams in Mersin Province, Turkey. Neurology. 2003;61(12):1804-1806.

4. Moghal S, Rajput AH, D’Arcy C, Rajput R. Prevalence of movement disorders in elderly community residents. Neuroepidemiology. 1994;13(4):175-178.

5. Louis ED. Understanding essential tremor: progress on the biological front. Curr Neurol Neurosci Rep. 2014;14(6):450.

6. Llinás R, Volkind RA. The olivo-cerebellar system: functional properties as revealed by harmaline-induced tremor. Exp Brain Res. 1973;18(1):69-87.

7. Deuschl G, Elble RJ. The pathophysiology of essential tremor. Neurology. 2000;54(11 suppl 4):S14-S2O.

8. Handforth A. Harmaline tremor: underlying mechanisms in a potential animal model of essential tremor. Tremor Other Hyperkinet Mov (NY). 2012;2:02-92-769-1.

9. Louis ED, et al. Neuropathological changes in essential tremor: 33 cases compared with 21 controls. Brain. 2007;130(pt 12):3297-3307.

10. Louis ED, Vonsattel JP. The emerging neuropathology of essential tremor. Mov Disord. 2008;23(2):174-182.

11. Yu M, et al. Increased number of Purkinje cell dendritic swellings in essential tremor. Eur J Neurol. 2012;19(4):625-630.

12. Sharifi S, Nederveen AJ, Booij J, van Rootselaar AF. Neuroimaging essentials in essential tremor: a systematic review. Neuroimage Clin. 2014;5:217-231.

13. Benito-León J, Labiano-Fontcuberta A. Linking essential tremor to the cerebellum: clinical evidence. Cerebellum. 2016;15(3):253-262.

14. Miwa H. Rodent models of tremor. Cerebellum. 2007;6(1):66-72.

15. Pan MK, Ni CL, Wu YC, Li YS, Kuo SH. Animal models of tremor: relevance to human tremor disorders. Tremor Other Hyperkinet Mov (N Y). 2018;8:587.

16. Kuo SH, et al. Current opinions and consensus for studying tremor in animal models. Cerebellum. 2019;18(6):1036-1063.

17. Kralic JE, et al. Genetic essential tremor in gamma-aminobutyric acidA receptor alpha1 subunit knockout mice. JClin Invest. 2005;115(3):774-779.

18. White JJ, et al. Pathogenesis of severe ataxia and tremor without the typical signs of neurodegeneration. Neurobiol Dis. 2016;86:86-98.

19. Pang ZP, et al. Synaptotagmin-2 is essential for survival and contributes to $\mathrm{Ca}^{2+}$ triggering of neurotransmitter release in central and neuromuscular synapses. J Neurosci. 2006;26(52):13493-13504.

20. Sun J, Pang ZP, Qin D, Fahim AT, Adachi R, Südhof TC. A dual-Ca ${ }^{2+}$-sensor model for neurotransmitter release in a central synapse. Nature. 2007;450(7170):676-682.

21. Luo F, Südhof TC. Synaptotagmin-7-mediated asynchronous release boosts high-fidelity synchronous transmission at a central synapse. Neuron. 2017;94(4):826-839.e3.

22. Fowler SC, et al. A force-plate actometer for quantitating rodent behaviors: illustrative data on locomotion, rotation, spatial patterning, stereotypies, and tremor. J Neurosci Methods. 2001;107(1-2):107-124.

23. Boecker $\mathrm{H}$, et al. The effect of ethanol on alcohol-responsive essential tremor: a positron emission tomography study. Ann Neurol. 1996;39(5):650-658.

24. Sommeijer JP, Levelt CN. Synaptotagmin-2 is a reliable marker for parvalbumin positive inhibitory boutons in the mouse visual cortex. PLOS One. 2012;7(4):e35323.

25. Südhof TC. Synaptotagmins: why so many? J Biol Chem. 2002;277(10):7629-7632.

26. Benabid AL, et al. Long-term suppression of tremor by chronic stimulation of the ventral intermediate thalamic nucleus. Lancet. 1991;337(8738):403-406.

27. Flora ED, Perera CL, Cameron AL, Maddern GJ. Deep brain stimulation for essential tremor: a systematic review. Mov Disord.
2010;25(11):1550-1559.

28. Deuschl G, Raethjen J, Hellriegel H, Elble R. Treatment of patients with essential tremor. Lancet Neurol. 2011;10(2):148-161.

29. Lein ES, et al. Genome-wide atlas of gene expression in the adult mouse brain. Nature. 2007;445(7124):168-176.

30. Haubensak W, et al. Genetic dissection of an amygdala microcircuit that gates conditioned fear. Nature. 2010;468(7321):270-276.

31. Chen S, Hillman DE. Compartmentation of the cerebellar cortex by protein kinase $\mathrm{C}$ delta. Neuroscience. 1993;56(1):177-188.

32. Hu H, Gan J, Jonas P. Interneurons. Fast-spiking, parvalbumin ${ }^{+}$GABAergic interneurons: from cellular design to microcircuit function. Science. 2014;345(6196):1255263.

33. Esposito MS, Capelli P, Arber S. Brainstem nucleus MdV mediates skilled forelimb motor tasks. Nature. 2014;508(7496):351-356

34. Ferreira-Pinto MJ, Ruder L, Capelli P, Arber S. Connecting circuits for supraspinal control of locomotion. Neuron. 2018;100(2):361-374.

35. Xu W, Südhof TC. A neural circuit for memory specificity and generalization. Science. 2013;339(6125):1290-1295.

36. Zhou M, Liu Z, Melin MD, Ng YH, Xu W, Südhof TC. A central amygdala to zona incerta projection is required for acquisition and remote recall of conditioned fear memory. Nat Neurosci. 2018;21(11):1515-1519.

37. Xu W, Morishita W, Buckmaster PS, Pang ZP, Malenka RC, Südhof TC. Distinct neuronal coding schemes in memory revealed by selective erasure of fast synchronous synaptic transmission. Neuron. 2012;73(5):990-1001.

38. Willner P. The validity of animal models of depression. Psychopharmacology (Berl). 1984;83(1):1-16.

39. Elble RJ, Schieber MH, Thach WT. Activity of muscle spindles, motor cortex and cerebellar nuclei during action tremor. Brain Res. 1984;323(2):330-334

40. Monzée J, Drew T, Smith AM. Effects of muscimol inactivation of the cerebellar nuclei on precision grip. J Neurophysiol. 2004;91(3):1240-1249. 
41. Paris-Robidas S, et al. Defective dentate nucleus GABA receptors in essential tremor. Brain. 2012;135(pt 1):105-116.

42. Brooks VB, Kozlovskaya IB, Atkin A, Horvath FE, Uno M. Effects of cooling dentate nucleus on tracking-task performance in monkeys. JNeurophysiol. 1973;36(6):974-995.

43. Cooke JD, Thomas JS. Forearm oscillation during cooling of the dentate nucleus in the monkey. Can J Physiol Pharmacol. 1976;54(4):430-436.

44. Xu J, Pang ZP, Shin OH, Südhof TC. Synaptotagmin-1 functions as a $\mathrm{Ca}^{2+}$ sensor for spontaneous release. Nat Neurosci. 2009;12(6):759-766.
45. Südhof TC. A molecular machine for neurotransmitter release: synaptotagmin and beyond. Nat Med. 2013;19(10):1227-1231.

46. Wolpert DM, Ghahramani Z, Jordan MI. An internal model for sensorimotor integration. Science. 1995;269(5232):1880-1882.

47. Boyden ES, Katoh A, Raymond JL. Cerebellumdependent learning: the role of multiple plasticity mechanisms. Annu Rev Neurosci. 2004;27:581-609.

48. Chen C, Arai I, Satterfield R, Young SM, Jonas P. Synaptotagmin 2 is the fast $\mathrm{Ca}^{2+}$ sensor at a central inhibitory synapse. Cell Rep. 2017;18(3):723-736.

49. Fujita H, Kodama T, du Lac S. Modular output cir- cuits of the fastigial nucleus for diverse motor and nonmotor functions of the cerebellar vermis. eLife. 2020;9:e58613.

50. Testa CM. Key issues in essential tremor genetics research: Where are we now and how can we move forward? Tremor Other Hyperkinet Mov (N Y). 2013;3:tre-03-105-1843-1.

51. Tio M, Tan EK. Genetics of essential tremor. Parkinsonism Relat Disord. 2016;22 (suppl1):S176-S178.

52. Zhao S, et al. Cell type-specific channelrhodopsin-2 transgenic mice for optogenetic dissection of neural circuitry function. Nat Methods. 2011;8(9):745-752. 